\title{
A non-linear rod model for folded elastic strips
}

\author{
Marcelo A. Dias * \\ School of Engineering, Brown University, Providence, Rhode Island 02912, USA
}

\author{
Basile Audoly ${ }^{\dagger}$ \\ UPMC Univ Paris 06,CNRS, UMR 7190, Institut Jean Le Rond d'Alembert, F-75005 Paris, \\ France
}

June 24, 2013

\begin{abstract}
We consider the equilibrium shapes of a thin, annular strip cut out in an elastic sheet. When a central fold is formed by creasing beyond the elastic limit, the strip has been observed to buckle out-ofplane. Starting from the theory of elastic plates, we derive a Kirchhoff rod model for the folded strip. A non-linear effective constitutive law incorporating the underlying geometrical constraints is derived, in which the angle the ridge appears as an internal degree of freedom. By contrast with traditional thinwalled beam models, this constitutive law captures large, non-rigid deformations of the cross-sections, including finite variations of the dihedral angle at the ridge. Using this effective rod theory, we identify a buckling instability that produces the out-of-plane configurations of the folded strip, and show that the strip behaves as an elastic ring having one frozen mode of curvature. In addition, we point out two novel buckling patterns: one where the centerline remains planar and the ridge angle is modulated; another one where the bending deformation is localized. These patterns are observed experimentally, explained based on stability analyses, and reproduced in simulations of the post-buckled configurations.
\end{abstract}

\section{Introduction}

Although the idea that a sheet of paper can be folded along an arbitrary curve is unfamiliar to many, performing this activity has been a form of art for quite some time. Bauhaus, the extinct German school of art and design, was a pioneer in developing the concept of curved folding structures by the end of the 1920s [1]. This practice often yields severely buckled and mechanically stiff sculptures featuring interesting structural properties and reveals new ways to think about engineering and architecture [2, 3, 4, Traditional origami has had a strong influence in the solution of many practical problems, to cite a few, the deployment of large membranes in space [5] and biomedical applications [6]. However, exploring this long established art form still has a lot of potential. Since the work by Huffman in 1976 [7], an elegant and groundbreaking description of the geometry of curved creases, more attention has been devoted to this subject [8, 9, 10, 11]. A mechanical approach of structures comprising curved creases has recently been proposed [12] motivated by the intriguing 3d shapes shown in figure 1. In the present paper, we build upon this recent work by further exploring the mechanical models governing folded structures.

Folded structures combine geometry and mechanics: they deform in an inextensible manner and their mechanics is constrained by the geometry of developable surfaces [13, 14]. Here, we consider one of the

\footnotetext{
*marcelo_dias@brown.edu

${ }^{\dagger}$ audoly@lmm.jussieu.fr
} 


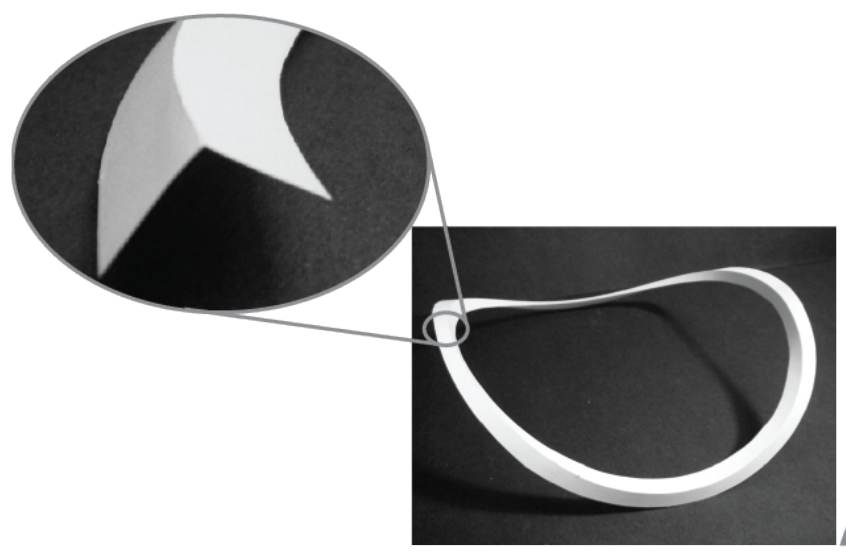

(a)

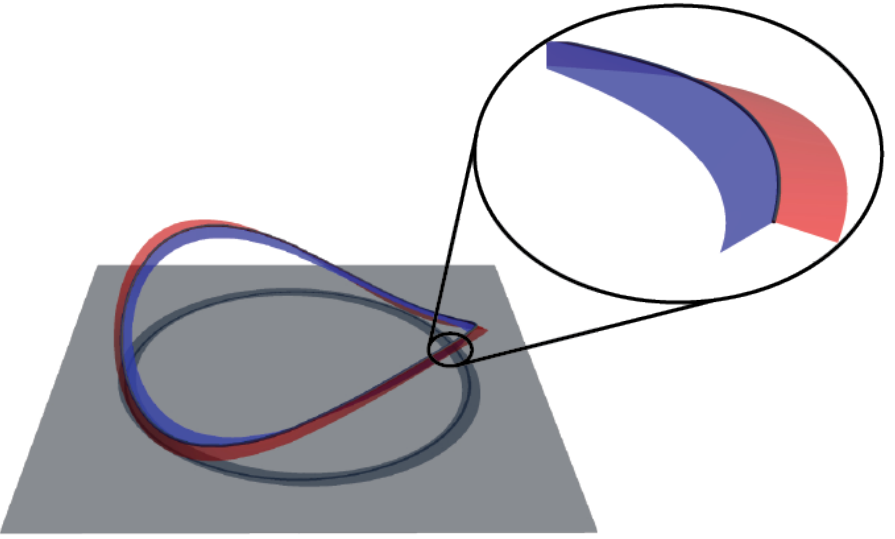

(b)

Figure 1: Buckling of an annular elastic strip having a central fold: (a) model cut out in an initially flat piece of paper; (b) one of the goals of this paper is to represent this folded strip as a thin elastic rod, the ridge angle being considered as an internal degree of freedom.

simplest folded structures: a narrow elastic plate comprising a central fold, as shown in figure 1. The role of geometry is apparent from the following observations, which anyone can reproduce with a paper model: the curvature of the crease line is minimum when the fold is flattened, a closed crease pattern results into a fold that buckles out of plane, while an open crease pattern results in a planar fold. These and other geometrical facts have been proved by Fuchs and Tabachnikov [9].

The mechanics of thin rods has a long history [15, 16, 17], and is used to tackle a number of problems from different fields today, such as the morphogenesis of slender objects [18, 19], the equilibrium shape of elongated biological filaments — such as DNA [20] and bacterial flagellum [21] — and the mechanics of the human hair [22, 23. The classical theory of rods, known as Kirchhoff's rod theory, assumes that all dimensions of the cross-section are comparable: the consequence is that the cross-sections of the rod deform almost rigidly as long as long as the strain remains small. This assumption does not apply to a folded strip: its cross-sections are slender, as shown in the inset of figure 1(a), and, as a result, they can bend by a large amount. In addition, the dihedral angle at the ridge can also vary by a large amount.

Vlasov's theory for thin-walled beams overcomes the limitations of Kirchhoff's theory by relaxing some kinematic constraints and considering additional modes of deformations of the cross-section. This kinematic enrichment can be justified from 3d elasticity: assuming a thin-walled geometry, asymptotic convergence of the $3 \mathrm{~d}$ problem to a rod model of Vlasov type has been established formally [24, 25]. This justification from 3d elasticity requires that the deformations are mild, however: the cross-sections can only bend by a small amount away from their natural shape.

Mechanical models have been proposed to capture the large deformations of thin-walled beams. The special case of curved cross-sections must be addressed starting from the theory of shell: in this case, the bending of the centerline involves a trade-off between the shell's bending and stretching energies [26, 27, [28, 29]. By contrast, the strip that we consider is developable; it can be studied based on an inextensible plate model, in which the stretching energy plays no role. A model for a thin elastic strip has been developed Sadowsky [30] in the case of a narrow ribbon, and later extended by Wunderlich to a finite width [31. These strip models have found numerous applications recently, see [32] for instance. They have been developed independently of the theory of rods, as they make use of unknowns that are tied to the developability constraint.

Here, we develop a unified view of strips and rods. We promote the viewpoint that elastic strips are just a special case of thin rods. The equations for the equilibrium of a narrow, inextensible plate are shown to be equivalent to those for an inextensible rod. To establish this equivalence, we identify the relevant geometrical constraints and derive of an effective, non-linear constitutive law. By doing so, we 


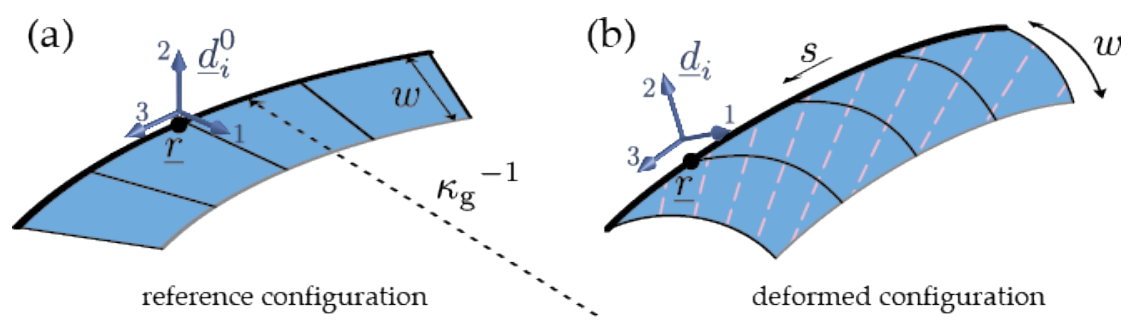

Figure 2: Analysis of a narrow elastic strip, without any fold. (a) One of the lateral boundaries is used as a centerline (thick curve), and its curvature in the reference configuration defines the geodesic curvature $\kappa_{\mathrm{g}}$. (b) The underlying mechanical model is an inextensible plate: in the deformed configuration, the generatrices, shown by the dashed lines, can make an arbitrary angle with the tangent $\underline{d}_{3}$ to the centerline. As a result, the cross-section (thin solid lines) can be significantly curved. The inextensibility constraint is used to reconstruct the mid-surface of the plate, based on the centerline shape $\underline{r}(s)$ (thick curve) and on the material frame $\underline{d}_{i}(s)$ : this allows the strip to be viewed as a thin rod.

extend Vlasov's models for thin-walled beams to large deformations. A unified perspective of strips and rods brings in the following benefits: instead of re-deriving the equations of equilibrium for strips from scratch, which is cumbersome, we show that the classical Kirchhoff equations are applicable; we identify for the first time the stress variables relevant to the strip model, which is crucial for stability problems; the extension of the strip model to handle natural (geodesic) curvature, or the presence of a central fold becomes straightforward, as we demonstrate; stability analyses and numerical solutions of post-buckled equilibria can be carried out in close analogy with what is routinely done for classical rods.

This paper is organized as follows. In section 2, we start by the smooth case, i.e. consider an elastic strip without a fold, and derive an equivalent rod model for it. In section 3, we extend this model to a folded strip, which we call a bistrip; this is one of the main results of our paper. In section 4, we derive circular solutions for the bistrip. Their stability is analyzed in section 5, and we identify two families of buckling modes: one family of modes explains the typical non-planar shapes of the closed bistrip reported earlier, while the second mode of buckling is novel. The predictions of the linear stability analysis are confronted to experiments in section 6, and to simulations of the post-buckled solutions in section 7 .

\section{Smooth case: equivalent rod model for a curved elastic strip}

We start by considering the case of a narrow strip having no central fold and show how it can be described using the language of thin elastic rods. The model we derive extends the model of Sadowsky [30] to account for the geodesic curvature of the strip and bridges the gap between his formulation and the classical theory of elastic rods.

\section{$2.1 \quad$ Kinematics and constraints}

We consider an inextensible elastic plate of thickness $h$ and width $w$ with a large aspect-ratio, $h \ll w$. In its undeformed configuration, the strip is planar. Under the action of a mechanical load, it is deformed into a $3 \mathrm{~d}$ shape, as sketched in figure 2. The deformed strip is parameterized by a space curve $\underline{r}(s)$, called the centerline, and by an orthonormal frame $\left(\underline{d}_{1}(s), \underline{d}_{2}(s), \underline{d}_{3}(s)\right)$, called the material frame. To define the centerline, we pick one of the lateral edges, which is the thick curve in the figure 2. The name 'centerline' is used for consistency with the theory of rods even though this curve is off the center of the strip. We denote by $s$ be the arc-length along this edge, and by $\underline{r}(s)$ the position in space of the centerline. The derivation with respect to arc-length is denoted by a prime. By definition, the tangent $\underline{r}^{\prime}(s)$ is a unit vector. Since the plate is inextensible, the arc-length $s$ will be used as a Lagrangian variable. The direct 
orthonormal frame $\underline{d}_{i}(s)$, with $i=1,2,3$, is defined in such a way that $\underline{d}_{3}$ is the tangent to the centerline,

$$
\underline{d}_{3}(s)=\underline{r}^{\prime}(s)
$$

and that $\underline{d}_{1}(s)$ and $\underline{d}_{3}(s)$ span the tangent plane to the midsurface of the elastic strip at the point $\underline{r}(s)$. Then, the unit vector $\underline{d}_{2}(s)=\underline{d}_{3}(s) \times \underline{d}_{1}(s)$ is normal to the midsurface at the edge $\underline{r}(s)$. By construction, the material frame is orthonormal and direct:

$$
\underline{d}_{i}(s) \cdot \underline{d}_{j}(s)=\delta_{i j},
$$

for any indices $i, j=\{1,2,3\}$ and for any $s$. Here, $\delta_{i j}$ denotes Kronecker's symbol, equal to 1 if $i=j$ and 0 otherwise.

The rate of rotation of the material frame with respect to the arc-length is captured by a vector $\underline{\omega}(s)$, which we call the Darboux vector or the twist-curvature strain. It is such that, for any $i=1,2,3$,

$$
\underline{d}_{i}^{\prime}(s)=\underline{\omega}(s) \times \underline{d}_{i}(s) .
$$

In classical rod theories, the rotation gradient $\underline{\omega}(s)$ measures the strain associated with the bending and twisting modes. Here, we use a plate model, and the bending strain is measured by the curvature form (second fundamental form) of the mid-surface, which we denote by $\underline{\underline{k}}$. Near a generic point $\underline{r}(s)$ on the centerline, we use the frame $\left(\underline{d}_{3}(s), \underline{d}_{1}(s)\right)$ tangent to surface: in this frame, the curvature tensor $\underline{\underline{k}}(s)$ is represented by a symmetric matrix,

$$
\underline{\underline{k}}(s)=\left(\begin{array}{ll}
k_{33}(s) & k_{13}(s) \\
k_{13}(s) & k_{11}(s)
\end{array}\right)_{\left(\underline{d}_{3}, \underline{d}_{1}\right)} .
$$

From the differential geometry of surfaces [13, 14, the gradient of the unit normal to a surface along any tangent direction can be computed from the second fundamental form. In particular, if we consider the gradient of the normal $\underline{d}_{2}(s)$ along the tangent $\underline{d}_{3}(s)$ to the centerline, we have $\underline{d}_{2}^{\prime}(s)=-\underline{\underline{k}}(s) \cdot \underline{d}_{3}(s)=$ $-\left(k_{13}(s) \underline{d}_{1}(s)+k_{33}(s) \underline{d}_{3}(s)\right)$. Identifying with the case $i=2$ equation (3), we find that the plate's bending strain and the equivalent rod's curvature strain are related by: $k_{13}(s)=\omega_{3}(s)$ and $k_{33}(s)=-\omega_{1}(s)$, where $\omega_{j}=\underline{\omega} \cdot \underline{d}_{j}$ denote the components of the Darboux vector in the material frame. We use this to express the second fundamental form of the midsurface of the plate, in terms of the Darboux vector $\underline{\omega}$ of the equivalent rod:

$$
\underline{\underline{k}}(s)=\left(\begin{array}{cc}
-\omega_{1}(s) & \omega_{3}(s) \\
\omega_{3}(s) & k_{11}(s)
\end{array}\right)_{\left(\underline{d}_{3}, \underline{d}_{1}\right)} .
$$

We assume that the midsurface of the plate is inextensible. This has two consequences. First, by Gauss' theorema egregium [13], its Gauss curvature, defined as the determinant of $\underline{\underline{k}}$, is zero:

$$
\mathcal{C}_{\mathrm{d}}\left(\underline{\omega}, k_{11}\right)=0, \quad \text { where } \mathcal{C}_{\mathrm{d}}\left(\underline{\omega}, k_{11}\right)=-\operatorname{det} \underline{\underline{k}}=\omega_{1} k_{11}+\left(\omega_{3}\right)^{2} .
$$

Second, we note that the quantity $\omega_{2}$, which defines the geodesic curvature of the centerline with respect to the midsurface, is conserved by isometries [13. Let $\kappa_{\mathrm{g}}=\omega_{2}^{0}$ denote the signed curvature of the edge (centerline) in the flat configuration of reference: in the actual configuration, the conservation of the geodesic curvature implies

$$
\mathcal{C}_{\mathrm{g}}(\underline{\omega})=0, \quad \text { where } \mathcal{C}_{\mathrm{g}}(\underline{\omega})=\kappa_{\mathrm{g}}-\omega_{2} .
$$

In equation (4), we have expressed the 'microscopic' strain $\underline{\underline{k}}$ in terms of the strain $\underline{\omega}$ of the equivalent rod, and of an additional 'internal' strain variable $k_{11}$. Equation (5) is a kinematic constraint. It could be used to eliminate the strain variable $k_{11}$ in favor of $\underline{\omega}$; we will refrain to do so, however, as this requires the additional assumption $\omega_{1} \neq 0$. Equation (6) is a second kinematic constraint applicable to the equivalent rod model. 


\subsection{Sadowsky's elastic energy}

Let us denote by $k^{*}$ the typical curvature of the plate, $|\underline{\underline{k}}| \sim k^{*}$. We assume that the strip is narrow, in the sense that the variations of the curvature tensor on distances comparable to the width $w$ remain small compared to $k^{*}: w|\underline{\nabla k}| \ll k^{*}$. Therefore, we ignore the dependence of the curvature strain on the transverse coordinate: in the entire cross-section containing the centerline point $\underline{r}(s)$, we approximate the curvature tensor by $\underline{\underline{k}}(s)$. This approximation has been used in the past to describe plates with straight centerlines [30]. It is possible to go beyond this approximation [31], as required when the curvature becomes large at localized spots along one of the edges [33] - we will refer to this model as a strip of finite width. An even more general model, applicable to strips of finite width and non-zero geodesic curvature, has been recently derived in [12].

We return to our small width approximation: the bending energy of the inextensible plate $E_{\mathrm{p}}$ can be integrated along the transverse direction, which yields

$$
E_{\mathrm{p}}=\int \frac{w D}{2}\left((1-\nu) \operatorname{tr}\left(\underline{\underline{k}}^{2}\right)+\nu \operatorname{tr}^{2} \underline{\underline{k}}\right) \mathrm{d} s,
$$

where $\left.D=E h^{3} /\left(12\left(1-\nu^{2}\right)\right)\right)$ is the bending modulus of the plate, $E$ its Young's modulus, $\nu$ its Poisson's ratio and $h$ its thickness. For a $2 \times 2$ matrix, the following identity holds: $\operatorname{tr}^{2} \underline{\underline{k}}=\operatorname{tr}\left(\underline{\underline{k}}^{2}\right)+2 \operatorname{det} \underline{\underline{k}}$. Dropping the determinant using the inextensibility condition (5), one can rewrite the elastic energy as

$$
E_{\mathrm{p}}=\int \frac{B}{2} \underline{\underline{k}}: \underline{\underline{k}} \mathrm{~d} s
$$

where $B=w D$ is a rod-type bending modulus, and the double contraction operator is defined by $\underline{\underline{a}}: \underline{\underline{b}}=\operatorname{tr}(\underline{\underline{a}} \cdot \underline{\underline{b}})=\sum_{i j} a_{i j} b_{j i}$. Note that when $k_{11}$ is eliminated using the constraint (5), the elastic energy $\bar{E}_{\mathrm{p}}$ coincides with that derived by Sadowsky [30]:

$$
E_{\mathrm{p}}=\frac{B}{2} \int\left(k_{33}^{2}+k_{11}^{2}+2{k_{13}}^{2}\right) \mathrm{d} s=\frac{B}{2} \int\left(\omega_{1}^{2}+2 \omega_{3}^{2}+\frac{\omega_{3}^{4}}{\omega_{1}^{2}}\right) \mathrm{d} s
$$

\subsection{Constitutive law}

We derive the equivalent rod model for our thin strip simply by viewing the energy $E_{\mathrm{p}}$ in equation (8) as the energy of a thin rod. The equivalent thin rod has one internal degree of freedom $k_{11}$ and is subjected to two kinematical constraints $\mathcal{C}_{\mathrm{d}}\left(\omega_{1}, \omega_{3}, k_{11}\right)$ and $\mathcal{C}_{\mathrm{g}}\left(\omega_{2}\right)$. In the $\mathrm{A}$, we derive the equations for a rod of this type. The condition of equilibrium of the internal variable reads

$$
-\frac{\delta E_{\mathrm{p}}}{\delta k_{11}}+B \lambda_{\mathrm{d}} \frac{\partial \mathcal{C}_{\mathrm{d}}}{\partial k_{11}}+B \lambda_{\mathrm{g}} \frac{\partial \mathcal{C}_{\mathrm{g}}}{\partial k_{11}}=0
$$

and the constitutive law as

$$
\underline{m}=\sum_{i=1}^{3}\left(\frac{\delta E_{\mathrm{p}}}{\delta \omega_{i}}-B \lambda_{\mathrm{d}} \frac{\partial \mathcal{C}_{\mathrm{d}}}{\partial \omega_{i}}-B \lambda_{\mathrm{g}} \frac{\partial \mathcal{C}_{\mathrm{g}}}{\partial \omega_{i}}\right) \underline{d}_{i} .
$$

Here, $\delta E_{\mathrm{p}} / \delta \omega_{i}$ and $\delta E_{\mathrm{p}} / \delta k_{11}$ denote the functional derivative of the elastic energy $E_{\mathrm{p}}$ with respect to the local strain $\omega_{i}(s)$ and internal variable $k_{11}(s)$, respectively. These equations were obtained by extending equations $87 \mathrm{a} 87 \mathrm{~b}$ of the appendix to the case of two constraints, and by identifying the internal degree of freedom $k=k_{11}$ and the energy $E_{\mathrm{el}}=E_{\mathrm{p}}$. The two Lagrange multipliers $\lambda_{\mathrm{d}}$ and $\lambda_{\mathrm{g}}$ are associated with the two constraint. For convenience, they have been rescaled with the bending modulus $B$, i.e. the quantity $\lambda$ in the appendix is replaced with $B \lambda_{\mathrm{d}}$ and $B \lambda_{\mathrm{g}}$.

Equation (9a) can be interpreted as the cancellation of the total generalized force acting on the internal variable, which is the sum of the standard force in the first term, and of constraint forces [34] in the last 
two terms. Similarly, the constitutive law in equation (9b) is made up of the usual contribution in rod theory, whereby the internal moment is the gradient of the elastic energy with respect to the twist and curvature strains, plus two other terms which are known as (generalized) constraint forces.

Using the explicit form of the energy $E_{\mathrm{p}}$ and of the constraints $\mathcal{C}_{\mathrm{d}}$ and $\mathcal{C}_{\mathrm{g}}$, equation (9a) yields

$$
k_{11}=\lambda_{\mathrm{d}} \omega_{1} .
$$

This equation will be used to eliminate the internal variable $k_{11}$ whenever it appears. In particular, the developability constraint in equation (5) takes the form

$$
\tilde{\mathcal{C}}_{\mathrm{d}}\left(\underline{\omega}, \lambda_{\mathrm{d}}\right)=0, \quad \text { where } \tilde{\mathcal{C}}_{\mathrm{d}}\left(\underline{\omega}, \lambda_{\mathrm{d}}\right)=\left(\omega_{1}\right)^{2} \lambda_{\mathrm{d}}+\left(\omega_{3}\right)^{2} .
$$

The second equation $(9 \mathrm{~b})$ yields

$$
\underline{m}=B\left(\omega_{1} \underline{d}_{1}+2 \omega_{3} \underline{d}_{3}\right)-B\left(\lambda_{\mathrm{d}}\left(k_{11} \underline{d}_{1}+2 \omega_{3} \underline{d}_{3}\right)-\lambda_{\mathrm{g}} \underline{d}_{2}\right) .
$$

Eliminating $k_{11}$ and projecting onto the material basis, we find the expressions of the twisting and bending moments $m_{i}=\underline{m} \cdot \underline{d}_{i}$ :

$$
\begin{aligned}
& m_{1}=B\left(1-\lambda_{\mathrm{d}}^{2}\right) \omega_{1} \\
& m_{2}=B \lambda_{\mathrm{g}} \\
& m_{3}=2 B\left(1-\lambda_{\mathrm{d}}\right) \omega_{3} .
\end{aligned}
$$

This is the constitutive law for a narrow elastic developable strip in the language of rods.

\subsection{Remarks}

The expression of the constraints in equations (6) and (11) and the constitutive law (12) are the main results of of section 2, They translate the inextensible strip model into the language of Kirchhoff's rods. Even though the strip is linearly elastic, its constitutive law is effectively non-linear because of the developability constraint.

We emphasize that the cross-sections of the strip are allowed to bend, as sketched in figure 2(b): this is required to preserve developabitility when the centerline is both twisted and bent, i.e. when both $\omega_{1}$ and $\omega_{3}$ are non-zero. We found that the strip is equivalent to an Euler-Bernoulli rod - for classical rods, the latter model is usually justified by assuming that the cross-sections do not bend but this is just a coincidence.

The strong formulation of the equilibrium of elastic rods is known as the Kirchhoff equations [22]. It can be derived by integration by parts from the principle of virtual work, as explained in A.

$$
\begin{aligned}
\underline{n}^{\prime}(s)+\underline{p}(s) & =\underline{0} \\
\underline{m}^{\prime}(s)+\underline{r}^{\prime}(s) \times \underline{n}(s)+\underline{q}(s) & =\underline{0} .
\end{aligned}
$$

Here, $\underline{n}$ is the Lagrange multiplier associated with the inextensibility and Euler-Bernoulli constraints, which can be interpreted as the internal force. Given a distribution of external force $p(s)$ and moment $\underline{q}(s)$, one can find the equilibria of the strip by solving the kinematical equations (1 -3$)$, the contraints (6) and (11), the constitutive law (12) and the equilibrium (13) for the unknowns $\underline{r}(s), \underline{d}_{i}(s), \omega_{i}(s), \lambda_{\mathrm{d}}(s)$ and $\lambda_{\mathrm{g}}(s)$.

\section{An equivalent rod model for the bistrip}

We now consider the case of an elastic strip having a central fold, as shown in figure 4. The central fold is represented by an elastic hinge. The flaps on both sides of the fold are represented using the mechanical model derived in section 2. We call bistrip this composite object, made up of the two flaps and the central fold. In this section, we derive an equivalent rod model for the bistrip, which takes into account both the bending stiffness of the inextensible flaps, and the stiffness of the central ridge. 


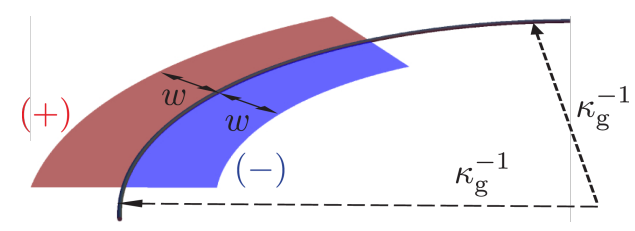

Figure 3: A strip with a central fold in its planar configuration, which we call a bistrip. The outer and inner flaps are labelled by $(+)$ and $(-)$, respectively. In this planar configuration, the curvature of the fold coincides with the geodesic curvature $\kappa_{\mathrm{g}}$.

(a)

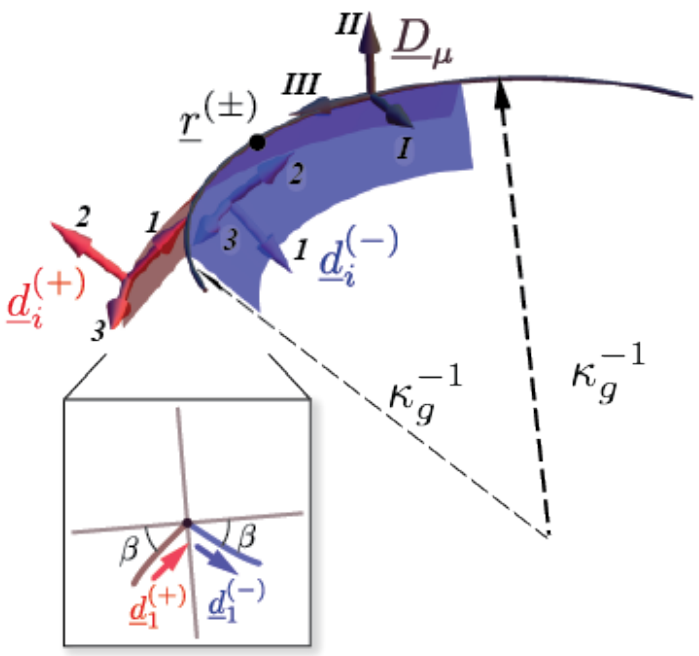

(b)
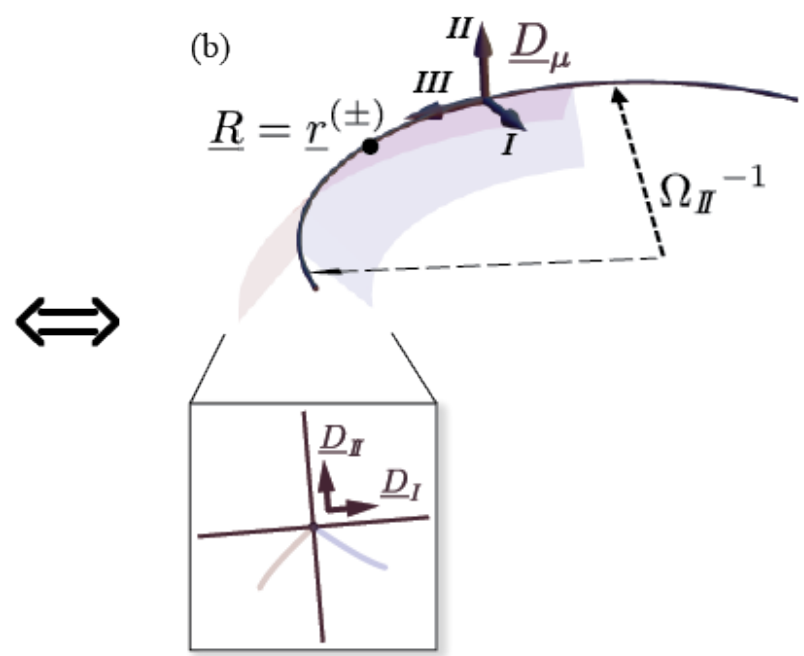

Figure 4: (a) A 3d configuration of the bistrip. The flaps $(+)$ and $(-)$ on both sides of the ridge are developable surface. Material frames $\underline{d}_{i}^{( \pm)}$are attached to them. (b) The equivalent rod model makes use of the common ridge as the centerline, $\underline{R}=\underline{r}$, and of the bisecting frame $\underline{D}_{\mu}$ as the material frame; the ridge angle $\beta$ is viewed as an internal degree of freedom. Note that the conserved geodesic curvature $\kappa_{\mathrm{g}}$ is measured along the tangent plane to the flaps, while the curvature strain $\Omega_{I I}$ of the centerline is measured in the plane spanned by $\underline{D}_{I}$ and $\underline{D}_{\mathbb{I I}}$. By equation (41b), $\Omega_{\mathbb{I}} \geq \kappa_{\mathrm{g}}$, and the ratio of these curvatures sets the ridge angle $\beta$.

\subsection{Kinematics}

The planar configuration of the bistrip is show in figure 3, in this configuration, the curvature of the central fold coincides with the geodesic curvature $\kappa_{\mathrm{g}}$. The outer and inner flaps, on each side of the central fold, are labelled by $(+)$ and $(-)$, respectively.

A typical $3 \mathrm{~d}$ configuration of the bistrip is shown in figure 4. To apply the analysis of section 2 to each of these flaps, we attach a material frame $\underline{d}_{i}^{(\epsilon)}$, with $i=1,2,3$ and $\epsilon= \pm$ to them. Let us denote by $\underline{R}(s)$ the common ridge, and $s$ the arc-length along this ridge. We use this ridge as the centerline for both flaps: in the notations of the previous section, $\underline{r}^{(+)}(s)=\underline{r}^{(-)}(s)=\underline{R}(s)$.

We observe that the tangent material vectors $\underline{d}_{3}^{( \pm)}$to both flaps are identical, since they share the same centerline: by equation $(1), \underline{d}_{3}^{(+)}(s)=\underline{r}^{(+)^{\prime}}(s)=\underline{R}^{\prime}(s)=\underline{r}^{(-)^{\prime}}(s)=\underline{d}_{3}^{(-)}(s)$. This allows us to define the bisecting frame $\underline{D}_{\mu}(s)$, with $\mu=I, I I$, III as follows. Let us first define $\underline{D}_{\mathbb{I I}}(s)$ to be the unit tangent to the ridge,

$$
\underline{R}^{\prime}(s)=\underline{D}_{I I I}(s),
$$

which coincides with the other tangents, $\underline{D}_{\mathbb{I I}}(s)=\underline{d}_{3}^{(+)}(s)=\underline{d}_{3}^{(-)}(s)$. The vector $\underline{D}_{I}(s)$ is defined as the unit vector that bisects the directions spanned by $\underline{d}_{1}^{(+)}(s)$ and $\underline{d}_{1}^{(-)}(s)$, as shown in the insets of figure 4 . 
Similarly, the vector $\underline{D}_{I}(s)$ is the unit vector that bisects the directions spanned by $\underline{d}_{2}^{(+)}(s)$ and $\underline{d}_{2}^{(-)}(s)$. By construction, the bisecting frame is an orthonormal frame,

$$
\underline{D}_{\mu}(s) \cdot \underline{D}_{\nu}(s)=\delta_{\mu \nu},
$$

for any pair of indices, $\mu, \nu=I, I I, I I I$. As a result, the gradient of rotation of the bisecting frame $\underline{D}_{\mu}$ can be measured by a Darboux vector $\underline{\Omega}$ such that

$$
\underline{D}_{\mu}^{\prime}(s)=\underline{\Omega}(s) \times \underline{D}_{\mu}(s) .
$$

The star symbols in equation labels, as in equations (14) and (16), will be used to mark any equation that defines the equivalent rod model.

Let us denote by $\beta$ half of the bending angle of the ridge, see the inset of figure 4 (a). This angle can be defined as a signed quantity if we use the orientation provided by the tangent $\underline{D} \mathbb{I I}=\underline{R}^{\prime}$ : by convention $\beta$ is positive in the figure. In terms of the parameter $\beta$, the dihedral angle at the ridge writes $\pi-2 \beta$.

The local material frames $\underline{d}_{i}^{(\epsilon)}(i=1,2,3$ and $\epsilon= \pm)$ can be reconstructed in terms of the bisecting frame and of the angle $\beta$ as follows:

$$
\begin{aligned}
& \underline{d}_{1}^{(\epsilon)}(s)=\underline{D}_{I}(s) \cos \beta(s)+\epsilon \underline{D}_{I I}(s) \sin \beta(s) \\
& \underline{d}_{2}^{(\epsilon)}(s)=-\epsilon \underline{D}_{I}(s) \sin \beta(s)+\underline{D}_{I I}(s) \cos \beta(s) \\
& \underline{d}_{3}^{(\epsilon)}(s)=\underline{D}_{I I I}(s) .
\end{aligned}
$$

By this equation, the entire bistrip can be reconstructed in terms of $\underline{R}, \underline{D}_{\mu}$ and $\beta$. Therefore, we use the ridge $\underline{R}$, the bisecting frame $\underline{D}_{\mu}$ and the ridge angle $\beta$ as the main unknowns. We shall show that the bistrip is equivalent to a rod having a centerline $\underline{R}(s)$ and a material frame $\underline{D}_{\mu}(s)$. The kinematic equations (14) shows that this equivalent rod is effectively an inextensible, Navier-Bernoulli rod — we emphasize that the cross-sections are not assumed to be rigid, however, as already discussed in section 2 . The rest of this section is concerned about deriving the constitutive law for the equivalent rod that captures the elasticity of the flaps and of the ridge.

\subsection{Reconstruction of local strains}

By differentiating equation (17) with respect to arc-length $s$, and identifying the result with equation (3) defining the local strains $\underline{\omega}^{(\epsilon)}$ in each flap, $\epsilon= \pm$, we obtain the following expression of the local strains:

$$
\underline{\omega}^{(\epsilon)}(s)=\underline{\Omega}(s)+\epsilon \beta^{\prime}(s) \underline{D}_{I I I}(s) .
$$

Note that the second term is associated with the 'internal' degree of freedom $\beta$ associated with the ridge angle.

To use the constitutive laws for a single strip derived earlier, we shall need the components of $\underline{\omega}^{(\epsilon)}$ in the local material frame, which we denote by $\omega_{i}^{(\epsilon)}=\underline{\omega}^{(\epsilon)} \cdot \underline{d}_{i}^{(\epsilon)}$ in the local frames. Projecting the previous equation, we find

$$
\begin{aligned}
& \omega_{1}^{(\epsilon)}(s)=\Omega_{I}(s) \cos \beta(s)+\epsilon \Omega_{I I}(s) \sin \beta(s) \\
& \omega_{2}^{(\epsilon)}(s)=-\epsilon \Omega_{I}(s) \sin \beta(s)+\Omega_{I I}(s) \cos \beta(s) \\
& \omega_{3}^{(\epsilon)}(s)=\Omega_{\text {III }}(s)+\epsilon \beta^{\prime}(s) .
\end{aligned}
$$

In the right-hand side, we denote by $\Omega_{\mu}=\underline{\Omega} \cdot \underline{D}_{\mu}$ the projections of the strain vector $\underline{\Omega}$ of the equivalent rod in its own material (bisecting) frame $\underline{D}_{\mu}$, with $\mu=I, I I, I I$. 


\subsection{Ridge: internal stress, constitutive law}

Let us denote by $\underline{p}(s)$ and $\underline{q}(s)$ the force and moment applied across the ridge, per unit arc-length $\mathrm{d} s$, by the inner region $(-)$ onto the outer region $(+)$. The outer flap feels a force $\underline{p}^{(+)}(s)=+\underline{p}(s)$ and moment $\underline{q}^{(+)}(s)=+\underline{q}(s)$. By the principle of action-reaction, the inner flap feels the opposite force and moment, $\underline{p}^{(-)}(s)=-\underline{p}(s)$ and moment $\underline{q}^{(-)}(s)=-\underline{q}(s)$. We write this in compact form as

$$
\begin{aligned}
& \underline{p}^{(\epsilon)}(s)=-\epsilon \underline{p}(s), \\
& \underline{q}^{(\epsilon)}(s)=-\epsilon \underline{q}(s),
\end{aligned}
$$

for $\epsilon= \pm$.

We model the central fold as an elastic hinge. The twisting moment $\underline{q}(s) \cdot \underline{R}^{\prime}(s)$ is therefore assumed to be a function of the angle $\beta$ :

$$
\underline{q}(s) \cdot \underline{R}^{\prime}(s)=Q_{\mathrm{r}}(2 \beta),
$$

where $Q_{\mathrm{r}}$ is the constitutive law of the ridge. By convention, the argument of $Q_{\mathrm{r}}$ is the turning angle $2 \beta$ at the ridge, and not $\beta$. This is motivated by the fact that the work done by the ridge is $(2 \delta \beta) Q_{\mathrm{r}}(2 \beta)$ when the parameter $\beta$ is incremented by $\delta \beta$.

Assuming that the constitutive law of the ridge is linear, we write

$$
Q_{\mathrm{r}}(2 \beta)=K_{\mathrm{r}} \times\left(2 \beta-2 \beta_{\mathrm{n}}\right)
$$

where $K_{\mathrm{r}}$ is the stiffness of the ridge, and $\beta_{\mathrm{n}}$ the natural value of the angle $\beta$. By creasing the strip, one induces irreversible deformations at the ridge: this is modeled by changing the value of $\beta_{\mathrm{n}}$.

\subsection{Equations of equilibrium}

Equation (13a) expresses the balance of force in each flap. With our current notations, it can be rewritten as $\underline{n}^{(\epsilon)^{\prime}}(s)+\underline{p}^{(\epsilon)}(s)=\underline{0}$, where $\epsilon= \pm$ labels the flaps and $\underline{n}^{(\epsilon)}$ denotes the internal force in each flap. We define the total internal force $\underline{N}(s)$ in the bistrip,

$$
\underline{N}(s)=\underline{n}^{(+)}(s)+\underline{n}^{(-)}(s) .
$$

By summing the local balance of forces and using the definition of $\underline{p}^{(\epsilon)}$ in equation $\sqrt{20}$, we find that the bistrip satisfies the global balance of forces

$$
\underline{N}^{\prime}(s)=\underline{0} .
$$

External force applied on the bistrip could be considered by adding a term in the left-hand side, as in the classical theory of rods.

Let us now turn to the balance of moments, which can be written in each flap as in equation $13 \mathrm{~b}$ ). Recalling that the two flaps share the same tangent $\underline{r}^{\prime}=\underline{R}^{\prime}=\underline{D}$ III , we have $\underline{m}^{(\epsilon)^{\prime}}+\underline{D}_{I I} \times \underline{n}^{(\epsilon)}+\underline{q}^{(\epsilon)}=\underline{0}^{\prime}$. In terms of the total moment $\underline{M}(s)$ in the bistrip, defined by

$$
\underline{M}(s)=\underline{m}^{(+)}(s)+\underline{m}^{(-)}(s)
$$

we write the global balance of moment in the bistrip as

$$
\underline{M}^{\prime}(s)+\underline{R}^{\prime}(s) \times \underline{N}(s)=\underline{0} .
$$

In equations 25) and (27), we have recovered the classical Kirchhoff equation for the equilibrium of thin rods: these equations express the global balance of forces and moments in the bistrip.

The global balance of moments (27) does not involve the internal twisting moment due to the ridge, $Q_{\mathrm{r}}$. A second equation for the balance of moments can be derived by projecting the local balance of 
moments in each strip onto the shared tangent: $\underline{m}^{(\epsilon)^{\prime}} \cdot \underline{R}^{\prime}+\underline{q}^{(\epsilon)} \cdot \underline{R}^{\prime}=0$. Subtracting the equations corresponding to $\epsilon=+$ and $\epsilon=-$, and expressing $\underline{q}^{( \pm)}$in terms of $Q_{\mathrm{r}}$ using equations 21 , and $(22)$, we have

$$
\underline{\Delta}^{\prime}(s) \cdot \underline{D}_{I I}(s)-Q_{\mathrm{r}}(2 \beta(s))=0,
$$

where $\Delta$ is half the difference of the internal moments:

$$
\underline{\Delta}(s)=\frac{\underline{m}^{(+)}(s)-\underline{m}^{(-)}(s)}{2} .
$$

As suggested by the presence of the ridge moment $Q_{\mathrm{r}}$, equation $(28)$ expresses the balance of moments at the ridge. It can be viewed as the equation that sets the internal degree of freedom $\beta$.

\subsection{Kinematic constraints applicable to the equivalent rod}

Two kinematic constraints are applicable in each flap $\epsilon= \pm$ : the geodesic constraint $\mathcal{C}_{\mathrm{g}}^{(\epsilon)}=0$ and the developability constraint $\mathcal{C}_{\mathrm{d}}^{(\epsilon)}=0$, see equations (5) and (6). Below, we express these constraints in terms of the centerline $\underline{R}$, of the bisecting frame $\underline{D}_{\mu}$, and of the ridge angle $\beta$. This yields effective kinematic constraints that are applicable to the equivalent rod.

Let us start by the geodesic constraint in equation (6). The geodesic curvature $\kappa_{\mathrm{g}}$ has been interpreted in figure 3, and is identical in both flaps: $\omega_{2}^{(+)}(s)=\omega_{2}^{(-)}(s)=\kappa_{\mathrm{g}}$. In particular, the average of the local curvature reads $\frac{1}{2}\left(\omega_{2}^{(-)}(s)+\omega_{2}^{(+)}(s)\right)=\kappa_{\mathrm{g}}$. Using equation $19 \mathrm{~b}$, we can rewrite the left-hand side in terms of the strain $\underline{\Omega}$ of the equivalent rod:

$$
\Omega_{I I}(s) \cos \beta(s)=\kappa_{\mathrm{g}} .
$$

By this kinematic constraint, the internal degree of freedom $\beta$ appears to be a function of the curvature strain $\Omega_{I I}$. We could eliminate $\beta$ in favor of $\Omega_{I I}$ using this equation. We shall instead view $\beta$ and $\Omega_{I I}$ as two degrees of freedom subjected to the constraint (30): this makes the final equations easier to interpret.

A second constraint follows from the equality $\omega_{2}^{(+)}(s)=\omega_{2}^{(-)}(s)$ : when expressed in terms of $\underline{\Omega}$ as above, it reads $\Omega_{I} \sin \beta=0$. We shall ignore the special case $\beta=0$ : as explained in section 4.2 , the bistrip is then on the boundary of the space of configurations and the equations of equilibrium are inapplicable anyway. Under the assumption $\beta \neq 0$, we have:

$$
\Omega_{I}(s)=0 .
$$

In view of the two constraints just derived, we can simplify the expressions of the local strains given earlier in equations (19a) and (19c):

$$
\begin{aligned}
& \omega_{1}^{(\epsilon)}(s)=\epsilon \Omega_{\text {II }}(s) \sin \beta(s) \\
& \omega_{3}^{(\epsilon)}(s)=\Omega_{\text {III }}(s)+\epsilon \beta^{\prime}(s) .
\end{aligned}
$$

The developability constraint in equation (11) can be simplified as well:

$$
\sigma^{2}(s) \lambda_{\mathrm{d}}^{(\epsilon)}(s)+\left(\Omega_{\text {III }}(s)+\epsilon \beta^{\prime}(s)\right)^{2}=0,
$$

where we have introduced an auxiliary variable $\sigma(s)=\epsilon \omega_{1}^{(\epsilon)}(s)$ which is given in terms of $\underline{\Omega}$ by

$$
\sigma(s)=\Omega_{\mathbb{I I}}(s) \sin \beta(s) .
$$

We note that the first term in equation (28) expressing the balance of moments at the ridge can be written in coordinates as: $\underline{\Delta}^{\prime} \cdot \underline{D}_{\mathbb{I I}}=\left(\underline{\Delta} \cdot \underline{D}_{\mathbb{I I}}\right)^{\prime}-\Delta \cdot \underline{D}_{\text {III }}^{\prime}=\Delta_{\mathbb{I I I}^{\prime}}-\underline{\Delta} \cdot\left(\underline{\Omega} \times \underline{D}_{\mathbb{I I}}\right)$, where $\underline{\Omega} \times \underline{D}_{\mathbb{I I}}=\Omega_{\mathbb{I I}} \underline{D}_{I}$ by the constraint in equation (31). Here, we denote by $\Delta_{\mu}=\underline{\Delta} \cdot \underline{D}_{\mu}$ and $\Omega_{\mu}=\underline{\Omega} \cdot \underline{D}_{\mu}$ the components of the differential internal moment $\underline{\Delta}$ and of the twist-curvature strain $\underline{\Omega}$ in the bisecting frame. We can therefore rewrite the equilibrium of the ridge as

$$
\Delta_{\text {III }}^{\prime}(s)-\Delta_{I}(s) \Omega_{I I}(s)-Q_{\mathrm{r}}(2 \beta(s))=0 .
$$




\subsection{Constitutive law}

To obtain a complete set of equations for the bistrip, we need the expressions of the total internal moment $\underline{M}$ and of the differential internal moment $\triangleq$ appearing in the equations of equilibrium. We derive the constitutive laws of the bistrip below, by combining the local constitutive law in each flap, and expressing them in terms of the twist-curvature strain $\underline{\Omega}$ of the effective rod.

Let us denote the average over the two flaps $\epsilon= \pm$ by angular brackets: $\left\langle f^{(\epsilon)}\right\rangle_{\epsilon}=\frac{1}{2}\left(f_{-}+f_{+}\right)$. In terms of the Lagrange multipliers $\lambda_{\mathrm{d}}^{(\epsilon)}$ associated with the developability constraint in each flap, we define the following quantities:

$$
\begin{aligned}
& b_{1}^{+}(s)=\left\langle 1-\left(\lambda_{\mathrm{d}}^{(\epsilon)}(s)\right)^{2}\right\rangle_{\epsilon} \\
& b_{1}^{-}(s)=\left\langle\epsilon\left(1-\left(\lambda_{\mathrm{d}}^{(\epsilon)}(s)\right)^{2}\right)\right\rangle_{\epsilon} \\
& b_{3}^{+}(s)=\left\langle 1-\lambda_{\mathrm{d}}^{(\epsilon)}(s)\right\rangle_{\epsilon} \\
& b_{3}^{-}(s)=\left\langle\epsilon\left(1-\lambda_{\mathrm{d}}^{(\epsilon)}(s)\right)\right\rangle_{\epsilon}
\end{aligned}
$$

Inserting the expression for $\lambda_{\mathrm{d}}^{(\epsilon)}$ found in equation 33 , we find explicit expressions for the auxiliary variables $b_{k}^{ \pm}$:

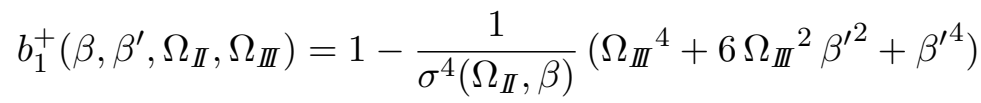

$$
\begin{aligned}
& b_{1}^{-}\left(\beta, \beta^{\prime}, \Omega_{\text {II }}, \Omega_{\text {III }}\right)=-\frac{4}{\sigma^{4}\left(\Omega_{\text {II }}, \beta\right)}\left(\Omega_{\text {III }}^{3} \beta^{\prime}+\Omega_{\text {III }} \beta^{\prime 3}\right) \\
& b_{3}^{+}\left(\beta, \beta^{\prime}, \Omega_{\mathbb{I I}}, \Omega_{\text {III }}\right)=1+\frac{1}{\sigma^{2}\left(\Omega_{\mathbb{I I}}, \beta\right)}\left(\Omega_{\mathbb{I I I}^{2}}+{\beta^{\prime 2}}^{2}\right) \\
& b_{3}^{-}\left(\beta, \beta^{\prime}, \Omega_{\mathbb{I I}}, \Omega_{\mathbb{I I}}\right)=\frac{2}{\sigma^{2}\left(\Omega_{\mathbb{I I}}, \beta\right)} \Omega_{\mathbb{I I}} \beta^{\prime} .
\end{aligned}
$$

The Lagrange multipliers $\lambda_{\mathrm{g}}^{(\epsilon)}$ associated with the geodesic constraints are eliminated in favor of their average $\Lambda_{+}(s)=\left\langle\lambda_{\mathrm{g}}^{(\epsilon)}(s)\right\rangle_{\epsilon}$ and half-difference $\Lambda_{-}(s)=\left\langle\epsilon \lambda_{\mathrm{g}}^{(\epsilon)}(s)\right\rangle_{\epsilon}$ : for $\epsilon= \pm$, they can be reconstructed by

$$
\lambda_{\mathrm{g}}^{(\epsilon)}(s)=\Lambda_{+}(s)+\epsilon \Lambda_{-}(s) .
$$

We view $\Lambda_{+}(s)$ and $\Lambda_{-}(s)$ as quantities attached to the equivalent rod: they are the Lagrange multipliers associated to the two kinematic constraints (30) and (31).

Let us now consider the local constitutive law in equation (12), which provides the expression of the internal moment $\underline{m}^{(\epsilon)}$ in each flap as a function of $\lambda_{\mathrm{d}}^{(\epsilon)}, \lambda_{\mathrm{g}}^{(\epsilon)}$ and $\omega_{2}^{(\epsilon)}$. These quantities can be expressed in terms of the properties of the equivalent rod, using equations (36), (38) and (6), respectively. This yields

$$
\underline{m}^{(\epsilon)}=B\left(\left(b_{1}^{+}+\epsilon b_{1}^{-}\right) \omega_{1}^{(\epsilon)} \underline{d}_{1}^{(\epsilon)}+\left(\Lambda_{+}+\epsilon \Lambda_{-}\right) \underline{d}_{2}^{(\epsilon)}+2\left(b_{3}^{+}+\epsilon b_{3}^{-}\right) \omega_{3}^{(\epsilon)} \underline{d}_{3}^{(\epsilon)}\right) .
$$

Inserting the expressions of the local strains $\omega_{1}^{(\epsilon)}$ and $\omega_{3}^{(\epsilon)}$ and of the local frame $\underline{d}_{i}^{(\epsilon)}$ in equations 32 , and (17), we obtain the following expressions of the internal moments $\underline{M}$ and $\underline{\Delta}$ defined in equations $(26)$ as 29$)$ :

$$
\left(\begin{array}{c}
M_{I} \\
M_{\text {II }} \\
M_{\text {III }} \\
\hline \Delta_{I} \\
\Delta_{\text {III }}
\end{array}\right)=B\left(\begin{array}{cc|cc|c}
\sin (2 \beta) b_{1}^{-} & 0 & 0 & -2 \sin \beta & 0 \\
2 \sin ^{2} \beta b_{1}^{+} & 0 & 2 \cos \beta & 0 & 0 \\
0 & 4 b_{3}^{+} & 0 & 0 & 4 b_{3}^{-} \\
\hline \cos \beta \sin \beta b_{1}^{+} & 0 & -\sin \beta & 0 & 0 \\
0 & 2 b_{3}^{-} & 0 & 0 & 2 b_{3}^{+}
\end{array}\right) \cdot\left(\begin{array}{c}
\Omega_{\text {II }} \\
\frac{\Omega_{\mathbb{I I}}}{\Lambda_{+}} \\
\Lambda_{-} \\
\hline \beta^{\prime}
\end{array}\right)
$$


This equation is the main result of section 3, and one of the main results of our paper. It yields the constitutive law of the rod that is equivalent to the bistrip, and captures the details of how the strip deforms at the 'microscopic' scale $w$. The constitutive law is geometrically exact and handles large deformations of the cross-section: the inextensibility of the strip is treated exactly, the cross-sections of the flaps may bend significantly, and the angle $\beta$ can change by a finite amount. The constitutive law is non-linear because of the geometry: the coefficients $b_{i}^{ \pm}\left(\beta, \beta^{\prime}, \Omega_{I I}, \Omega_{\mathbb{I I}}\right)$ in the matrix above depend non-linearly on the strains through equations (37). By contrast, the underlying plate model makes use of a linear constitutive law: the bending energy of the plate is quadratic with respect to the strain, see equation (7).

This constitutive law $(39)$ depends on the bending strain $\Omega_{\mathbb{I}}$ — recall that the other bending mode $\Omega_{I}$ is frozen by equation (31) — and on the twisting strain $\Omega_{\mathbb{I I}}$, like in the classical theory of thin elastic rods. It also depends on the internal degree of freedom $\beta$ and on its derivative $\beta^{\prime}$, and on the Lagrange multipliers $\Lambda_{+}$and $\Lambda_{-}$associated with the two applicable constraints.

The expression of $\Delta_{I I}$ has been omitted in the constitutive law (39), as it does not appear in the equations of equilibrium: it is absent from equation (35) expressing the balance of moments at the ridge. For reference, its expression is $\Delta_{I I}(s)=\sin ^{2} \beta(s) b_{1}^{-}\left(\beta(s), \Omega_{I I}(s), \Omega_{I I I}(s), \beta^{\prime}(s)\right) \Omega_{I I}(s)+\cos \beta(s) \Lambda_{-}(s)$.

\subsection{Summary: effective rod model for a bistrip}

We recapitulate the equations that govern the bistrip, collecting them by family: these are all the equations that we have marked by a star symbol so far.

- The main unknowns of the model are $(i)$ the centerline $\underline{R}(s)$ and a direct orthonormal frame $\left(\underline{D}_{\mu}(s)\right)_{\mu=1,2,3}$, as usual in rod theory; and $(i i)$ an 'internal' degree of freedom, namely the ridge angle $\beta(s)$.

- The kinematic equation defining the strain $\underline{\Omega}$ reads

$$
\underline{D}_{\mu}^{\prime}(s)=\underline{\Omega}(s) \times \underline{D}_{\mu}(s) .
$$

This is the standard definition of the twist-curvature strain vector for rods.

- The following kinematic constraints are applicable:

$$
\begin{gathered}
\underline{R}^{\prime}(s)=\underline{D}_{I I I}(s), \\
\Omega_{I I}(s) \cos \beta(s)=\kappa_{\mathrm{g}}, \\
\Omega_{I}(s)=0 .
\end{gathered}
$$

Equation 41a defines the classical inextensible Euler-Bernoulli rod model: $s$ being a Lagrangian variable, $\left|\underline{R}^{\prime}(s)\right|=\left|D_{\text {III }}(s)\right|=1$ implies inextensibility and $\underline{R}^{\prime} \cdot \underline{D}_{\mu}=\underline{D}_{\text {III }} \cdot \underline{D}_{\mu}=0$ for $\mu=I, I I$ implies the absence of normal shear. The two kinematic constraints $41 \mathrm{~b} 41 \mathrm{c}$ are specific to the bistrip.

- The equations of equilibrium read

$$
\begin{gathered}
\underline{N^{\prime}}(s)=\underline{0} \\
\underline{M^{\prime}}(s)+\underline{R}^{\prime}(s) \times \underline{N}(s)=\underline{0} \\
\Delta_{\text {III }}^{\prime}(s)-\Delta_{I}(s) \Omega_{I I}(s)-Q_{\mathrm{r}}(2 \beta(s))=0
\end{gathered}
$$

Equations (42a 42b) are the classical Kirchhoff equations for rods. The additional equation (42c) expresses the balance of moments at the ridge, and sets the equilibrium value of the internal degree of freedom $\beta$. 
- Finally, the constitutive law reads

$$
\left(\begin{array}{c}
M_{I} \\
M_{\text {II }} \\
M_{\text {III }} \\
\hline \Delta_{I} \\
\Delta_{\text {III }}
\end{array}\right)=B\left(\begin{array}{cc|cc|c}
\sin (2 \beta) b_{1}^{-} & 0 & 0 & -2 \sin \beta & 0 \\
2 \sin ^{2} \beta b_{1}^{+} & 0 & 2 \cos \beta & 0 & 0 \\
0 & 4 b_{3}^{+} & 0 & 0 & 4 b_{3}^{-} \\
\hline \cos \beta \sin \beta b_{1}^{+} & 0 & -\sin \beta & 0 & 0 \\
0 & 2 b_{3}^{-} & 0 & 0 & 2 b_{3}^{+}
\end{array}\right) \cdot\left(\begin{array}{c}
\Omega_{\text {II }} \\
\Omega_{\text {III }} \\
\hline \Lambda_{+} \\
\Lambda_{-} \\
\hline \beta^{\prime}
\end{array}\right),
$$

where the secondary variables $b_{k}^{ \pm}\left(\beta, \beta^{\prime}, \Omega_{\mathbb{I I}}, \Omega_{\mathbb{I I}}\right)$, with $k=1,3$, were defined in equation (37).

\subsection{Simplified constitutive law for nearly circular configurations}

A much simpler version of the constitutive law can be derived, which is applicable to near circular geometries. We assume that the ridge angle and the twist can be expanded as:

$$
\begin{aligned}
\Omega_{\text {III }}(s) & =0+\Omega_{\text {III }}^{1}(s)+\cdots \\
\beta(s) & =\beta_{0}+\beta_{1}(s)+\cdots
\end{aligned}
$$

Here, $\beta_{0}$ is a constant, to be specified later, and $\beta_{1}(s)$ and $\Omega_{\text {III }}^{1}(s)$ are assumed to be small. Until the end of this section, we retain the linear terms $\beta_{1}(s)$ and $\Omega_{\text {III }}^{1}(s)$, but neglect higher-order terms.

Later on, we shall show that the expansions in equation (44) are applicable to the analysis of circular configurations of the bistrip, and of their stability.

When inserting the expansions in to equation (37), we find

$$
b_{1}^{+}=1, \quad b_{3}^{+}=1, \quad b_{1}^{-}=0, \quad b_{3}^{-}=0,
$$

up to second-order terms: almost all the highly non-linear terms disappear from the constitutive law a few of them are still present as we do not assume the angle $\beta_{0}$ to be small.

To linear order, the constitutive law for $\Delta_{I}$ reads

$$
\Delta_{I}(s)=B \sin \beta(s)\left(\Omega_{I}(s) \cos \beta(s)-\Lambda_{+}(s)\right)=B \sin \beta(s)\left(\kappa_{\mathrm{g}}-\Lambda_{+}(s)\right)
$$

after using the geodesic constraint in equation (41b). Inserting the constitutive law $\Delta_{\text {III }}(s)=2 B \beta_{1}^{\prime}(s)$ into the balance of moments at the ridge in equation (42c), inserting the above expression of $\Delta_{I}(s)$ into the resulting expression, and solving for $\Lambda_{+}(s)$, we find

$$
\Lambda_{+}(s)=\kappa_{\mathrm{g}}+\frac{\frac{1}{B} Q_{\mathrm{r}}(2 \beta(s))-2 \beta^{\prime \prime}(s)}{\Omega_{I I}(s) \sin \beta(s)} .
$$

Inserting this expression into the constitutive law 43 for $\underline{M}$, and retaining terms up to the linear order, we find

$$
\begin{aligned}
M_{I}(s) & =\Lambda_{I}(s) \\
M_{\text {II }}(s) & =M_{\text {II }}^{\text {nc }}\left(\Omega_{I I}(s), \beta(s), \beta^{\prime \prime}(s)\right) \\
M_{\text {III }}(s) & =4 B \Omega_{\text {III }}(s) .
\end{aligned}
$$

Here, $M_{I I}^{\text {nc }}$ denotes the constitutive law in bending, in the nearly circular case:

$$
M_{I I}^{\mathrm{nc}}\left(\Omega_{I I}, \beta, \beta^{\prime \prime}\right)=2 B \Omega_{I I}+\frac{2 B}{\Omega_{I I} \tan \beta}\left(\frac{1}{B} Q_{\mathrm{r}}(2 \beta)-2 \beta^{\prime \prime}\right) .
$$

In equation (46a), we have denoted by $\Lambda_{I}(s)=-2 B \sin \beta(s) \Lambda_{-}(s)$ the right-hand side. This $\Lambda_{I}(s)$ is viewed as the Lagrange multiplier associated with the constraint $\Omega_{I}=0$, and is used in replacement 
of the other unknown $\Lambda_{-}$. Note that $\beta$ can be viewed in equation $46 \mathrm{~b}$ as a function of $\Omega_{I I}$ through the constraint (41b): because of the presence of the factor $\beta^{\prime \prime}, M_{I I}$ depends on the first and second derivatives of $\Omega_{I I}$ as well, and the constitutive law is of the second-gradient type.

The constitutive law (46) is considerably simpler than the fully non-linear one in equation (43). It incorporates the equilibrium of the ridge: there is no need to use equation (42c) when we use the above constitutive law. This effective rod model resembles a classical rod model: the twist mode is governed by a classical linear constitutive law, $M_{\text {III }}=4 B \Omega_{\mathbb{I I}}$; the internal degree of freedom $\beta$ can be viewed a function of the bending strain $\Omega_{I I}$ by the constraint $41 \mathrm{~b}$ ); the constitutive law in bending is non-linear and of second-gradient type, see equation $46 \mathrm{~d}$ ); the curvature $\Omega_{I}=0$ is frozen and the corresponding bending moment $M_{I}=\Lambda_{I}$ is a Lagrange multiplier.

As we shall show, this simple constitutive is applicable to the analysis of the circular solutions in section 4, and to their stability in section 5. To compute the post-buckled solution in section 7, however, we shall revert to the fully non-linear constitutive law of the previous section.

\section{Circular solutions}

The rest of the paper is concerned with the analysis of the equilibria of a bistrip closed into a loop, see figure 1. This problem has been considered recently in [12]: the authors have shown that the planar configuration of the bistrip is non-planar as it buckles into a $3 \mathrm{~d}$ shape. They observed that for very small widths $\left(\kappa_{\mathrm{g}} w \ll 1\right)$ the dihedral angle is unaffected by this buckling instability, and remains uniform and constant in the post-buckled regime. Revisiting this problem, we show that $(i)$ it is a variant of the classical buckling analysis of an elastic ring, (ii) a simple expression for the buckling threshold can be derived, (iii) the conservation of the dihedral angle in the post-buckled regime can be explained based on symmetry considerations, and $(i v)$ the closed bistrip can exhibit another, novel type of buckling instability.

\subsection{Preparation of the circular configuration}

We start by explaining in this section how the initial, circular state of the bistrip is prepared. The procedure is sketched in figure 5, and we invite the reader to perform the following experiments himself or herself:

- An annular region of size $2 w$ and mean radius $\rho$ is cut out in a piece of paper. This sets the value of the geodesic curvature to

$$
\kappa_{\mathrm{g}}=\frac{1}{\rho} .
$$

- Next, a sector of angle $\gamma$ is removed, see figure 5(a). The ridge is formed by creasing along the central circle, as shown in figure 5(b). This amounts to reset the value of the natural ridge angle $\beta_{\mathrm{n}}$ appearing in the constitutive law (23) to a non-zero value. As a result of this, the ridge angle takes on a value $\beta=\beta^{\dagger}$ which is set by the the competition of the ridge energy (which is minimum when $\beta_{\mathrm{n}}$ ) and of the bending energy of the flaps (which is minimum when $\beta=0$ ).

- Finally, the free ends are glued together, as shown in figure 5(c). The arc-length of the ridge is $L=(2 \pi \rho-\gamma) \rho$, and so the radius of curvature is

$$
r_{0}=\frac{L}{2 \pi}=\left(1-\frac{\gamma}{2 \pi}\right) \rho
$$

We analyze the circular configuration using the notations in figure 6 the $z$-axis is normal to the loop, and the origin of coordinates is at the center of the circular ridge. We use the polar coordinates $(r, \varphi)$ 


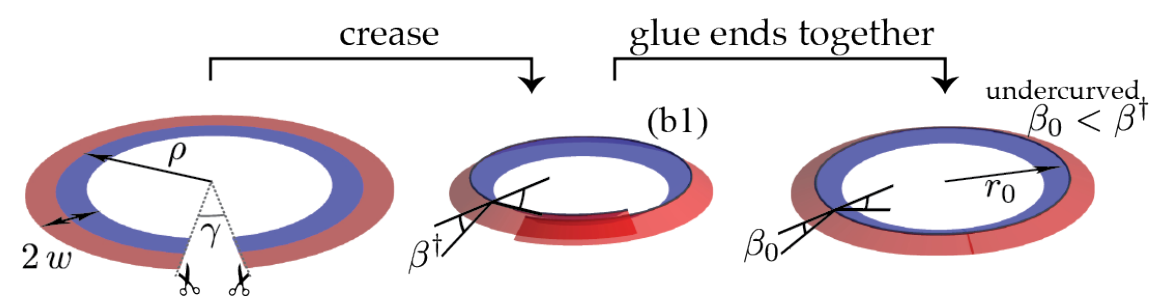

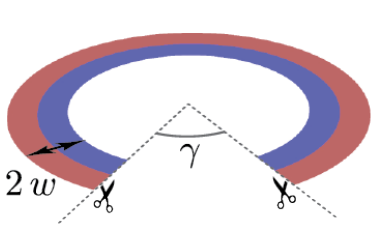

(a)

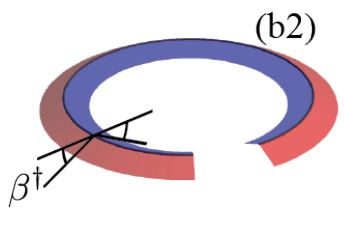

(b)

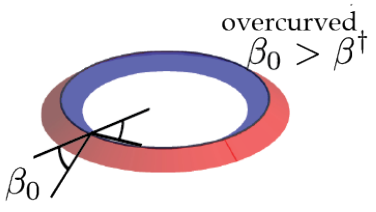

(c)

Figure 5: The circular bistrip is prepared by the following steps: (a) an annular region is cut out in a flat piece of paper, and a sector of angle $\gamma$ is removed from it. (b) The ridge is creased: in the equations, this amounts to set the natural ridge angle $\beta_{\mathrm{n}}$ to a non-zero value. As a result, the ridge angle $\beta$ has a non-zero equilibrium value $\beta^{\dagger}$ and the curvature of the centerline increases. Depending on the value of $\gamma$, this can leads to an overlap (b1), or to a residual gap (b2). (c) The bistrip is closed up by bringing the endpoints together. Note that the circular configuration may be unstable, as studied later.

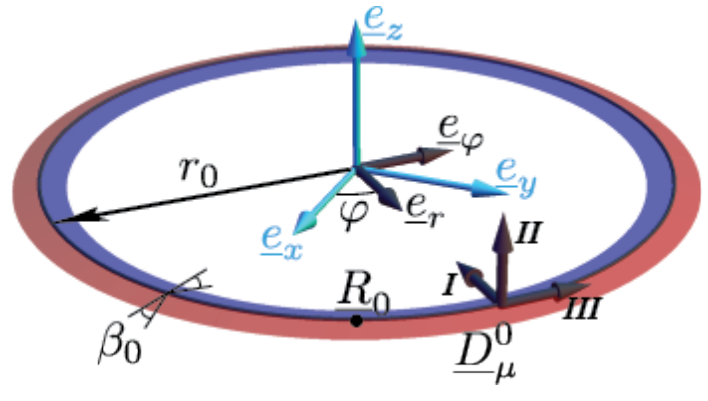

Figure 6: Geometry of a circular configuration.

in the plane $(x, y)$ containing the ridge, and denote the polar basis by $\underline{e}_{r}(\varphi)=\cos \varphi \underline{e}_{x}+\sin \varphi \underline{e}_{y}$ and $\underline{e}_{\varphi}(\varphi)=-\sin \varphi \underline{e}_{x}+\cos \varphi \underline{e}_{y}$. Then, the centerline reads

$$
\underline{R}_{0}(s)=r_{0} \underline{e}_{r}\left(\frac{s}{r_{0}}\right)
$$

and the material frame

$$
\underline{D}_{I}^{0}(s)=-\underline{e}_{r}\left(\frac{s}{r_{0}}\right), \quad \underline{D}_{I I}^{0}(s)=\underline{e}_{z}, \quad \underline{D}_{I I I}^{0}(s)=\underline{e}_{\varphi}\left(\frac{s}{r_{0}}\right)
$$

By the definition of the twist-curvature strain $\underline{\Omega}$ in equation $(16)$, we have

$$
\underline{\Omega}_{0}=\Omega_{I I}^{0} \underline{e}_{z}, \quad \text { where } \Omega_{I I}^{0}=\frac{1}{r_{0}} .
$$

This is compatible with the constraint $\Omega_{I}^{0}=\underline{\Omega}^{0} \cdot \underline{D}_{I}^{0}=0$. The twist is also zero, $\Omega_{\mathrm{III}}^{0}=0$. The ridge angle $\beta_{0}$ can be found by equation (30) and it is uniform:

$$
\beta_{0}=\cos ^{-1}\left(\frac{\kappa_{\mathrm{g}}}{\Omega_{\text {II }}^{0}}\right)=\cos ^{-1}\left(1-\frac{\gamma}{2 \pi}\right) .
$$


Note that we can orient the $z$ axis so that it points in the same direction as the ridge, as in figure 5 where both the axis and the ridge point upwards. Then, the angle is in the range

$$
0 \leq \beta_{0}<\frac{\pi}{2}
$$

\subsection{Stress in the circular configuration}

Since $\beta(s)=\beta_{0}$ is constant and $\Omega_{\mathbb{I I}}(s)=0$ cancels, the expansion postulated in equation (44) holds, with $\beta_{1}(s)=0$ and $\Omega_{\mathbb{I I}}^{1}(s)=0$. Therefore, the simplified constitutive law in equation 46 is applicable:

$$
\begin{aligned}
M_{I}^{0}(s) & =\Lambda_{I} \\
M_{I I}^{0}(s) & =M_{I I}^{\text {nc }}\left(\frac{1}{r_{0}}, \beta_{0}, 0\right) \\
M_{\text {III }}^{0}(s) & =0 .
\end{aligned}
$$

We seek a cylindrically symmetric solution, and the Lagrange multiplier $\Lambda_{I}$ is therefore assumed to be constant.

By the balance of forces in equation (42a), the internal force is constant, $\underline{N}_{0}(s)=\underline{N}_{0}$. By the balance of moments in equation (42b), we find that $\Lambda_{I}$ and $\underline{N}_{0}$ both cancel. Therefore, the internal stress in the circular bistrip reads:

$$
\begin{aligned}
& \underline{N}^{0}(s)=\underline{0} \\
& \underline{M}^{0}(s)=M_{\text {II }}^{0} \underline{e}_{z},
\end{aligned}
$$

where the internal bending moment reads

$$
M_{I I}^{0}=M_{I I}^{\mathrm{nc}}\left(\frac{1}{r_{0}}, \beta_{0}, 0\right)=\frac{2 B}{r_{0}}\left(1+\frac{1}{\tan \beta_{0}} \frac{Q_{0} r_{0}^{2}}{B}\right) .
$$

Here, we have introduced the shorthand notation $Q_{0}$ for the ridge moment in the circular state:

$$
Q_{0}=Q_{\mathrm{r}}\left(2 \beta_{0}\right)
$$

The first contributions to $M_{I I}^{0}$ in equation (55) is proportional to $B$ and comes from the elasticity of the flaps. The second term, proportional to $Q_{0}$, comes from the elasticity of the ridge.

The sign of the internal bending moment $M_{\text {II }}^{0}$ is crucial for the stability of the ring. It can be positive or negative, depending on how much the ridge has been creased (term $Q_{0}$ ) and how large the ridge angle is (term $\beta_{0}$ ). Following reference [35], the circular solution is said to be undercurved when the internal moment $M_{\text {II }}^{0}$ tends to increase its curvature, and overcurved in the opposite case:

$$
\begin{cases}M_{I I}^{0}<0 & : \text { undercurved } \\ M_{I}^{0}>0 & : \text { overcurved }\end{cases}
$$

The undercurved case corresponds to figure 5(b1): before they are glued together, the ends are overlapping; to close up the ring, one has to decrease its curvature below its natural value, making the ring wider and flatter: $\beta_{0}<\beta^{\dagger}$. The overcurved case corresponds to the figure 5(b2): before the ends of the ring are glued together, they are separated by a gap: closing up the ends involves decreasing the curvature below its natural value, making the ring narrower and the ridge angle larger, $\beta_{0}>\beta^{\dagger}$.

We note that the stress in equation (54) becomes singular when $\beta_{0}=0$. To avoid this difficulty, we shall assume

$$
\beta_{0} \neq 0
$$


The case $\beta_{0}=0$ is pathological because the circular solution then sits on the boundary of the space of admissible configuration, not in the interior. Indeed, the ridge curvature $\Omega_{I I}$ is at its maximum value, $\kappa_{\mathrm{g}}$ : this curvature cannot vary to first order, and as a result of this the in-plane projection of the ridge deforms rigidly. The equations of equilibrium that we derived are not applicable in this special case. To study the case $\beta_{0}=0$, we would need to relax constraints and consider an extensible plate model.

\section{$5 \quad$ Linear stability of the circular solutions}

In reference [12, non-planar configurations of the closed bistrip were observed using a paper model; postbuckled solutions were also calculated, showing striking similarities with the experimental patterns. A typical picture of a paper model is reproduced in our figure 1.

Here, we show that these shapes are produced by a buckling instability affecting the circular solutions of section 4. Our model allows us to identify the stress that causes this instability: this is simply the prestress in equation (54). The eigenmodes and the buckling threshold are calculated analytically. The selection of the azimuthal wavenumber (number of bumps) is explained. A second family mode of buckling is pointed out, and demonstrated experimentally.

\subsection{Parameterization of the buckling modes}

The buckling modes are parameterized by three functions, $\left\{\hat{\psi}_{I}(s), \hat{\psi}_{I I}(s), \hat{\psi}_{\mathbb{I I I}}(s)\right\}$, which are the components in the undeformed basis of the infinitesimal rotation vector,

$$
\underline{\hat{\psi}}(s)=\sum_{\mu=I}^{\text {III }} \hat{\psi}_{\mu}(s) \underline{D}_{\mu}^{0}(s),
$$

where hats denote perturbations, i.e. small increments. The infinitesimal rotation $\underline{\hat{\psi}}(s)$ is used to reconstruct the perturbed material frame by

$$
\underline{D}_{\mu}(s)=\underline{D}_{\mu}^{0}(s)+\underline{\hat{\psi}}(s) \times \underline{D}_{\mu}^{0}(s) .
$$

The perturbed centerline can then be found by integration of the equation $\underline{R}^{\prime}=\underline{D}_{\mathbb{I I}}$, up to a constant of integration which is an important rigid-body translation.

\subsection{Infinitesimal perturbation to the twist and curvature}

To compute the perturbed strain vector $\underline{\Omega}$, we take the derivative of equation $(60)$ and use equation (16): $\underline{\Omega}(s) \times \underline{D}_{\mu}(s)=\underline{\Omega}_{0}(s) \times\left(\underline{D}_{\mu}(s)-\underline{\hat{\psi}}(s) \times \underline{D}_{\mu}^{0}(s)\right)+\underline{\hat{\psi}}^{\prime}(s) \times \underline{D}_{\mu}^{0}(s)+\underline{\hat{\psi}}(s) \times\left(\underline{\Omega}_{0}(s) \times \underline{D}_{\mu}^{0}(s)\right)$. Rearranging the terms and using Jacobi's identity, we derive the following expression for the perturbation $\underline{\hat{\Omega}}(s)=$ $\underline{\Omega}(s)-\underline{\Omega}_{0}(s)$ to the strain vector:

$$
\underline{\hat{\Omega}}(s)=\underline{\hat{\psi}^{\prime}}(s)-\underline{\Omega}_{0}(s) \times \underline{\hat{\psi}}(s) .
$$

This equation expresses the geometric compatibility of the increment of rotation $\hat{\psi}(s)$ and of strain $\underline{\hat{\Omega}}(s)$, and is well-known, see [36] and [22, eq. 3.51] for instance. Its right-hand side can be interpreted as the co-moving derivative of $\underline{\hat{\psi}}$ in the undeformed material frame $\underline{D}_{\mu}^{0}$, which shows that

$$
\underline{\hat{\Omega}}(s)=\sum_{\mu=I}^{\text {III }} \hat{\psi}_{\mu}^{\prime}(s) \underline{D}_{\mu}^{0}(s) .
$$


We denote by $\hat{\Omega}_{\mu}(s)$ the first-order perturbation to the strain caused by the perturbation:

$$
\hat{\Omega}_{\mu}(s)=\Omega_{\mu}(s)-\Omega_{\mu}^{0}(s)=\underline{\Omega}(s) \cdot \underline{D}_{\mu}(s)-\underline{\Omega}_{0}(s) \cdot \underline{D}_{\mu}^{0}(s) .
$$

Neglecting second-order terms, we can write this variation of a product as $\hat{\Omega}_{\mu}=\underline{\hat{\Omega}} \cdot \underline{D}_{\mu}^{0}+\underline{\Omega}_{0} \cdot\left(\underline{\hat{\psi}} \times \underline{D}_{\mu}^{0}\right)$. The first is given by equation 62 , and we have

$$
\hat{\Omega}_{\mu}(s)=\hat{\psi}_{\mu}^{\prime}(s)+\underline{\Omega}_{0}(s) \cdot\left(\underline{\hat{\psi}}(s) \times \underline{D}_{\mu}^{0}(s)\right) .
$$

Inserting the expression of $\underline{\Omega}_{0}$ in equation $(51)$, this yields

$$
\begin{aligned}
& \hat{\Omega}_{I}(s)=\hat{\psi}_{I}^{\prime}(s)+\frac{1}{r_{0}} \hat{\psi}_{\text {III }}(s) \\
& \hat{\Omega}_{I I}(s)=\hat{\psi}_{\text {II }}^{\prime}(s) \\
& \hat{\Omega}_{\text {III }}(s)=\hat{\psi}_{\text {III }}^{\prime}(s)-\frac{1}{r_{0}} \hat{\psi}_{I}(s)
\end{aligned}
$$

The curvature $\Omega_{I}(s)$ being frozen by equation $\left.41 \mathrm{c}\right)$, its perturbation is zero, $\hat{\Omega}_{I}(s)=0$. The rotation $\hat{\psi}_{\text {III }}$ can then be eliminated from equation 65a):

$$
\hat{\psi}_{\text {III }}(s)=-r_{0} \hat{\psi}_{I}^{\prime}(s) .
$$

Inserting into equation $65 \mathrm{c}$, we find

$$
\hat{\Omega}_{I I I}(s)=-\frac{1}{r_{0}}\left(r_{0}^{2} \hat{\psi}_{I}^{\prime \prime}(s)+\hat{\psi}_{I}(s)\right) .
$$

\subsection{Azimuthal wavenumber}

Given the cylindrical invariance of the base solution, we seek buckling modes that depend harmonically on the polar variable $\varphi=\frac{s}{r_{0}}=\Omega_{I I}^{0} s$. These buckling modes are indexed by an integer wavenumber $n \geq 0$,

$$
\left(\hat{\psi}_{I}(s), \hat{\psi}_{\text {II }}(s), \hat{\psi}_{\text {III }}(s)\right)=\left(\hat{\Psi}_{I}, \hat{\Psi}_{\text {II }}, \hat{\Psi}_{\text {III }}\right) e^{\frac{i n s}{r_{0}}}
$$

The coefficients in the parenthesis in the right-hand side are the complex amplitudes of the infinitesimal rotation.

By a classical argument, the cases $n=0$ and $n=1$, which correspond to rigid-body rotations, are ruled out. Indeed, when $n=0, \hat{\Omega}_{I}=0$ by the constraint, $\hat{\Omega}_{I I}=0$ by equation $(65 \mathrm{~b})$, and $\hat{\Omega}_{\text {III }}$ is proportional to $\hat{\psi}_{I}$ by equation $(67)$; the condition that the centerline closes up after one turn requires that the constant value of $\hat{\psi}_{I}$ is zero. As a result, all the strain components $\Omega_{\mu}$ stay unperturbed when $n=0$, which corresponds to a rigid-body motion of the bistrip. A similar argument shows that $n=1$ corresponds to a rigid-body motion of the bistrip as well. Therefore, we only consider azimuthal wavenumbers $n$ in the buckling analysis such that

$$
n \geq 2 \text {. }
$$

\subsection{Linearized equilibrium}

Linearizing the balance of forces $42 \mathrm{a}$, we have $\underline{\hat{N}}^{\prime}(s)=\underline{0}$, and so the perturbation to the internal force is a constant vector, $\underline{\hat{N}}(s)=\underline{0}$. We know $\underline{N}_{0}(s)=\underline{0}$ from equation (54a), and therefore the total internal force reads $\underline{N}(s)=\underline{\hat{N}}$ to first order in the perturbation. Inserting into the balance of moments $42 \mathrm{c}$ and retaining first order terms, we find

$$
\underline{\hat{M}}^{\prime}(s)=-\underline{D}^{0}(\mathrm{II}) \times \underline{\hat{N}} .
$$


Since we study buckling modes that are pure Fourier modes, the components of the vector $\underline{M}^{\prime}(s)$ in the unperturbed frame $\underline{D}_{\mu}^{0}$ all depend on $s$ as $\exp \left(\frac{i n s}{r_{0}}\right)$ with $n \geq 2$. By contrast, the components of the right-hand side $\left(-\underline{D}_{\mathbb{I I}}^{0}(s) \times \underline{\hat{N}}\right)$ in the unperturbed frame $\underline{D}_{\mu}^{0}$, which can be computed explicitly, have only two non-zero Fourier components, with wavevectors 0 and 1 . Therefore, we conclude that both sides of equation 70 must cancel: $\underline{\hat{M}^{\prime}}(s)=\underline{0}$. Integrating, we find that $\underline{\hat{M}}(s)$ is a constant. For the components of $\underline{\hat{M}}(s)$ in the material frame to be harmonic with $n \geq 2$, this constant must in fact be zero:

$$
\underline{\hat{M}}(s)=\underline{0} .
$$

We have just shown that the buckling modes leave the internal force and moment constant, to first order.

\subsection{Linearized constitutive law}

In equation (63), we have assumed that the bending and twist strain can be expanded as $\Omega_{\mu}(s)=$ $\Omega_{\mu}^{0}(s)+\hat{\Omega}_{\mu}(s)$, with $\mu=\mathbb{I}$, III. Using the unperturbed strain $\Omega_{\mu}^{0}(s)$ given earlier in equation $(51)$, this yields

$$
\begin{aligned}
& \Omega_{I I}(s)=\Omega_{I I}^{0}+\hat{\Omega}_{I I}(s) \\
& \Omega_{\text {III }}(s)=0+\hat{\Omega}_{\text {III }}(s) .
\end{aligned}
$$

Using the geodesic constraint in equation $(41 \mathrm{~b})$, we can derive the expansion for the ridge angle $\beta$, from that of $\Omega_{\mathbb{I}}$ :

$$
\beta(s)=\beta_{0}+\hat{\beta}(s)
$$

where

$$
\hat{\beta}(s)=\frac{r_{0}}{\tan \beta_{0}} \hat{\Omega}_{\mathbb{I I}}(s) .
$$

Comparison of equations $72 \mathrm{~b} 72 \mathrm{c}$ with equations $44 \mathrm{a} 44 \mathrm{~b}$ shows that the linear stability analysis involves exactly the type of expansion that was postulated in section 3.8, which we dubbed the 'nearly circular' case. Therefore, we can use the simplified constitutive law (46) in the linear stability analysis. Linearizing this constitutive law, we get

$$
\begin{aligned}
& \hat{M}_{I I}=\frac{\partial M_{I I}^{\mathrm{nc}}\left(\Omega_{I I}^{0}, \beta_{0}, 0\right)}{\partial \Omega_{I I}} \hat{\Omega}_{I I}+\frac{\partial M_{I I}^{\mathrm{nc}}\left(\Omega_{I I}^{0}, \beta_{0}, 0\right)}{\partial \beta} \hat{\beta}+\frac{\partial M_{I I}^{\mathrm{nc}}\left(\Omega_{I I}^{0}, \beta_{0}, 0\right)}{\partial \beta^{\prime \prime}} \hat{\beta}^{\prime \prime} \\
& \hat{M}_{\text {III }}=4 B \hat{\Omega}_{I I I}
\end{aligned}
$$

Note that we have not included the linearized constitutive for $\hat{M}_{I}$, as we are not interested in reconstructing the Lagrange multiplier $\hat{\Lambda}_{I}$. The quantities in the left-hand side are the perturbations to the strain components $\hat{M}_{\mu}=\underline{M} \cdot \underline{D}_{\mu}-\underline{M}_{0} \cdot \underline{D}_{\mu}^{0}$. The perturbation to the internal moment $\underline{\hat{M}}$ can be written in terms of them as

$$
\begin{aligned}
\hat{M}(s)=\underline{M}(s)-\underline{M}_{0}(s) & =\sum_{\mu=I}^{\mathbb{I I}}\left(\hat{M}_{\mu}(s)+M_{\mu}^{0}\right) \underline{D}_{\mu}(s)-M_{\mu}^{0} \underline{D}_{\mu}^{0}(s) \\
& =\sum_{\mu=I}^{I I I} \hat{M}_{\mu}(s) \underline{D}_{\mu}(s)+\sum_{\mu=I}^{I I} M_{\mu}^{0} \underline{\psi}(s) \times \underline{D}_{\mu}^{0}(s)=\sum_{\mu=I}^{\text {III }} \hat{M}_{\mu}(s) \underline{D}_{\mu}^{0}(s)+\underline{\hat{\psi}}(s) \times \underline{M}^{0},
\end{aligned}
$$

after dropping second-order terms. By the balance of moments in equation (71), the left-hand side is zero. Inserting the expression of $\underline{M}_{0}$ in equation $(54 \mathrm{~b})$ in the right-hand side, and projecting onto the directions $\mu=\mathbb{I}$ and $\mu=\mathbb{I I I}$, we find

$$
\begin{aligned}
\hat{M}_{\text {II }}(s) & =0 \\
\hat{M}_{\text {III }}(s)+M_{\text {II }}^{0} \hat{\psi}_{I}(s) & =0 .
\end{aligned}
$$




\subsection{Centerline mode}

Eliminating $M_{\text {III }}$ from equations $73 \mathrm{~b}$ and $75 \mathrm{~b}$ and inserting the expression of $\underline{\Omega}_{\mathbb{I I}}$ obtained by the kinematic equation (67), we find an eigenvalue problem for the periodic function $\hat{\psi}_{I}$ :

$$
-\frac{M_{I I}^{0} \hat{\psi}_{I}(s)}{4 B}=-\frac{1}{r_{0}}\left(r_{0}^{2} \hat{\psi}_{I}^{\prime \prime}(s)+\hat{\psi}_{I}(s)\right) .
$$

Inserting the harmonic dependence on the arc-length $s$ given in equation (68), this leads to

$$
\left(\frac{M_{I I}^{0} r_{0}}{4 B}+\left(n^{2}-1\right)\right) \hat{\Psi}_{I}=0
$$

When the factor in parentheses cancels, non-zero values of the rotation $\hat{\Psi}_{I}$ are possible. This corresponds to a family of buckling modes which we call the centerline mode.

In equation (77), equating the factor in parenthesis to zero yields the critical value of the bending prestress $M_{\text {II }}^{0}$ where the ridge mode occurs. The prestress $M_{I I}^{0}$ is always negative when the parenthesis cancels in equation (77), since $n \geq 2$. Therefore, the centerline buckling is only possible in the undercurved case.

This prestress is itself a function of the natural ridge angle $\beta_{\mathrm{n}}$ by equation (55). Inserting this function, we find an equation for the critical value of the natural ridge angle $\beta_{\mathrm{n}}=\beta_{\mathrm{n}, \text { crit }}^{\text {ctl }}\left(\bar{K}_{\mathrm{r}}, \beta_{0}, n\right)$ where the centerline buckling mode occurs:

$$
\bar{K}_{\mathrm{r}} \frac{2 \beta_{0}-2 \beta_{\mathrm{n}, \mathrm{crit}}^{\mathrm{ctl}}\left(\bar{K}_{\mathrm{r}}, \beta_{0}, n\right)}{\tan \beta_{0}}=1-2 n^{2}, \quad n \geq 2 .
$$

This $\beta_{\mathrm{n}, \text { crit }}^{\text {ctl }}\left(\bar{K}_{\mathrm{r}}, \beta_{0}, n\right)$, is a function of the initial ridge angle $\beta_{0}$, of the wavenumber $n$, and of the dimensionless ridge stiffness

$$
\bar{K}_{\mathrm{r}}=\frac{r_{0}^{2} K_{\mathrm{r}}}{B} .
$$

The mode can be reconstructed by picking an infinitesimal value of $\hat{\Psi}_{I}$ in equation (68), and by computing $\underline{\hat{\psi}}$ by equation $(66)$. The twisting strain is then given by equation (67). On the other hand, the bending strain $\Omega_{I}$, which is proportional to $\hat{\psi}_{I I}$ remains zero: the centerline mode involves buckling in pure twist. By equation (72c), the ridge angle remains unperturbed as well - this is why we call it a centerline mode. The mode can be visualized by reconstructing the perturbed material frame by equation (60), and by integrating along the tangent to find the deformed centerline. In figure 7 the first two centerline modes, $n=2$ and 3 , are visualized.

In reference [12], post-buckled configurations of the centerline mode have been observed both in experiments using paper model and in simulations. Here, we have shown that this mode occurs by an instability very similar to classical instabilities for elastic rings [37, 38, 19], and have calculated the buckling threshold analytically. Our equation predicts that $n=2$ is the first unstable mode, when the prestress $M_{I I}^{0}$ is made more and more negative.

\subsection{Analysis of the ridge mode}

We now proceed to analyze the second family of buckling modes, by using the remaining equations derived in the beginning of this section. Combining the balance of moments $75 \mathrm{a}$ ) and the linearized constitutive law 73a), we find a relation between $\hat{\Omega}_{\mathbb{I I}}, \hat{\beta}$ and $\hat{\beta}^{\prime \prime}$ :

$$
\hat{\beta}^{\prime \prime}=\frac{K_{\mathrm{r}}-Q_{0} \csc \left(2 \beta_{0}\right)}{B} \hat{\beta}+\frac{1}{2 r_{0}}\left(\tan \beta_{0}-\frac{r_{0}^{2} Q_{0}}{B}\right) \hat{\Omega}_{I I} .
$$




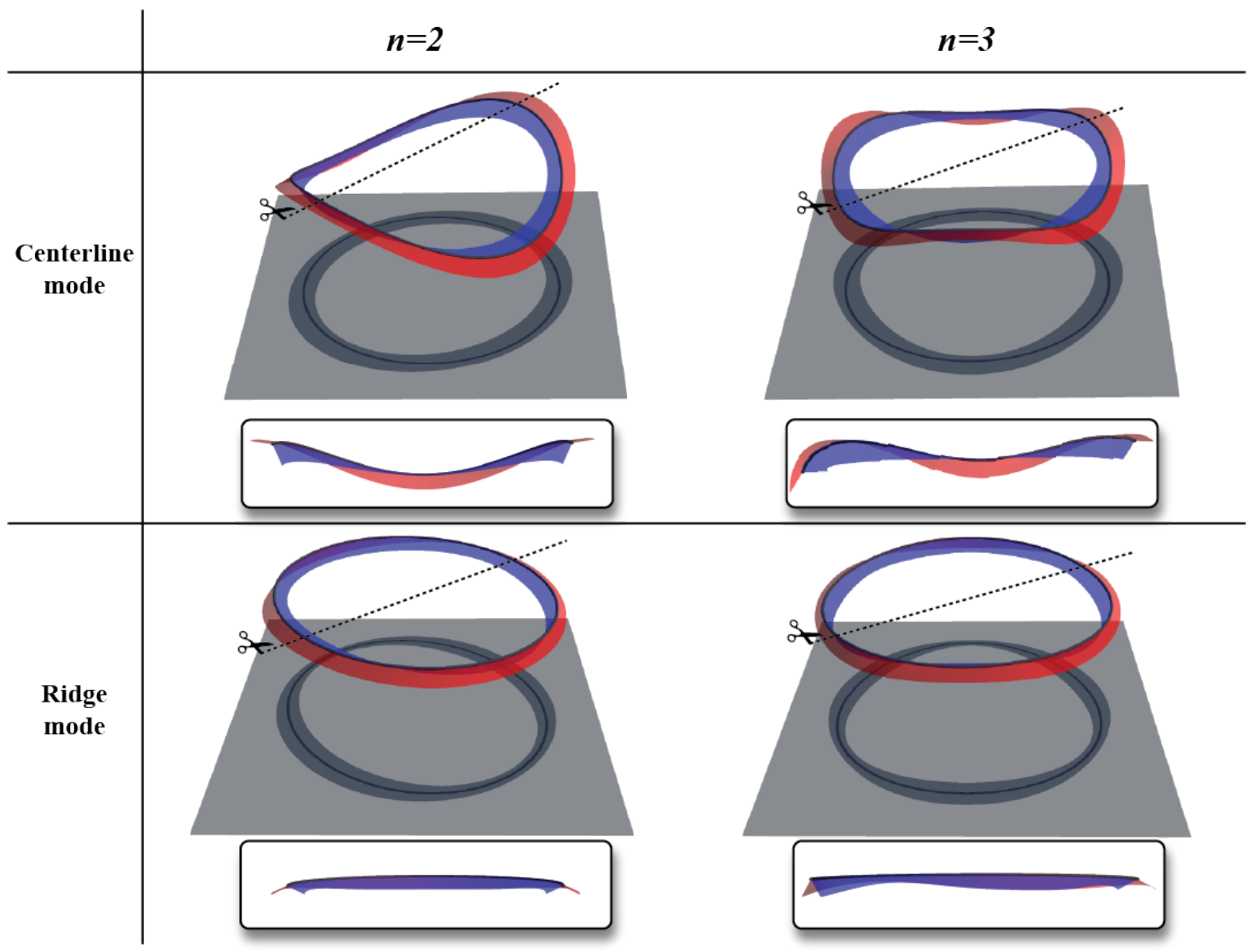

Figure 7: The two families of buckling modes for an initially circular configuration: centerline and ridge modes. In each family, the first two modes are shown, corresponding to the wavenumbers $n=2$ and 3 . In the shadows obtained by projection onto a normal plane, the centerline appears to be oscillating from one edge to the other in the centerline mode, but appears centered in the ridge mode. Cuts along the dashed line are shown in the framed insets. In the centerline mode, which occurs in pure twist, the central ridge goes out of plane, the dihedral angle is conserved and the cross-sections swing back and forth about the centerline. By contrast, the ridge mode involves a modulation of the dihedral angle, and the central ridge stays planar.

Then, we eliminate $\hat{\beta}$ using the linearized geodesic constraint in equation $(72 \mathrm{~d})$ and the kinematic equation $65 \mathrm{~b}$, and obtain an eigenvalue problem for the periodic function $\hat{\psi}_{I I}$ :

$$
\hat{\psi}_{I I}^{\prime \prime \prime}(s)=\frac{1}{r_{0}^{2}}\left[\frac{\tan ^{2}\left(\beta_{0}\right)}{2}+\bar{K}_{\mathrm{r}}-\frac{1}{2}\left(\tan \beta_{0}+\frac{1}{\sin \beta_{0} \cos \beta_{0}}\right)\left(2 \beta_{0}-2 \beta_{\mathrm{n}}\right) \bar{K}_{\mathrm{r}}\right] \hat{\psi}_{I I}^{\prime}(s) .
$$

The harmonic dependence on the arc-length given in equation (68) is used again to solve equation (81). As earlier, this yields the equation for the critical value of the natural angle $\beta_{\mathrm{n}}$ at the ridge, which we denote by $\beta_{\mathrm{n}, \mathrm{crit}}^{\text {rige }}\left(\bar{K}_{\mathrm{r}}, \beta_{0}, n\right)$ :

$$
\bar{K}_{\mathrm{r}} \frac{2 \beta_{0}-2 \beta_{\mathrm{n}, \text { crit }}^{\text {ridge }}\left(\bar{K}_{\mathrm{r}}, \beta_{0}, n\right)}{\tan \beta_{0}}=\frac{\bar{K}_{\mathrm{r}}+n^{2}+\frac{\tan ^{2} \beta_{0}}{2}}{\frac{1}{2}+\tan ^{2} \beta_{0}}
$$

The left-hand side of this equation is the second term in the right-hand side of equation (55). This shows that the residual stress $M_{I I}^{0}$ is always positive at the onset of bifurcation: the ridge buckling instability occurs in the overcurved case. 
The reconstruction of the ridge mode is similar to that of the centerline mode. The ridge mode only involves bending $\Omega_{\mathbb{I I}}$, and the twist remains zero, $\Omega_{\mathbb{I I}}=0$ : the ridge mode is a pure bending mode. A consequence of this is that the centerline remains planar. The first two ridges modes $(n=2$ and 3$)$ are shown in figure 7. By equation (82), the first unstable ridge mode is the one with $n=2$ bumps.

This ridge mode has not been discussed earlier in the literature, to the best of our knowledge.

\subsection{Interpretation of the buckling modes by a symmetry argument}

We have found two families of buckling modes: the centerline mode, and the ridge mode. Each buckling mode can occur with an arbitrary azimuthal wavenumber, indexed by an integer $n \geq 2$. The centerline modes occur in pure twist: the twist $\Omega_{\mathbb{I I}}$ is non-zero, making the central ridge go out of plane, while the unconstrained curvature $\Omega_{\mathbb{I I}}$ remains unchanged, implying that the ridge angle $\beta$ remains unperturbed. By contrast, the ridge mode occurs in pure bending: the twist $\Omega_{\text {III }}$ remains zero, making the central ridge remain planar, while the unconstrained curvature $\Omega_{I I}$ is modulated together with the ridge angle $\beta$.

These features of the buckling modes can be interpreted based on symmetry considerations. In $B$, we identify a symmetry of the equilibrium equations for the bistrip, which leaves the circular base state invariant. The two families of modes that we have obtained are the eigenvectors of this symmetry. Indeed, the eigenvector corresponding to the eigenvalue +1 satisfies $\tilde{\Omega}_{\text {III }}=+\Omega_{\text {III }}$ which, in view of equation 92 in the appendix, implies $\Omega_{\mathbb{I I}}=0$ : this is the ridge mode. The eigenvector corresponding to the eigenvalue -1 satisfies $\tilde{\Omega}_{\mathbb{I}}=-\Omega_{\mathbb{I}}$, implying $\Omega_{\mathbb{I I}}=0$ : this is the centerline mode. This symmetry explains why the eigenvalue problems for the centerline and ridge modes in equations (76) and (81) are uncoupled, and why the ridge angle $\beta$ is unaffected by the ridge mode, as observed in previous work [12].

\section{Experiments}

\subsection{Experimental buckling modes}

We confront the stability analysis carried out in the previous section to experimental pictures of paper models. An annular region is cut out in a piece of paper; as explained earlier in figure 5, an angular sector of size $\gamma$ is removed, see (a) in figure 8, which sets the dihedral angle $\beta_{0}$ of the circular solution by equation (52); the permanent deformations involved in pleating the ridge in step (b) amount to change the natural value $\beta_{\mathrm{n}}$ of the ridge angle in the constitutive law. The circular configuration is not observed, as the bistrip buckles. The top row (a1-c1) in figure 8 corresponds to the undercurved case: a buckling mode with $n=2$ bumps is observed, as already reported in [12. The features of the centerline predicted by the linear stability analysis are confirmed: the deformation involves twist, the centerline becomes non-planar and the ridge angle remains uniform.

The second row $(\mathrm{a} 2-\mathrm{c} 2)$ in figure 8 shows the overcurved case, i.e. when $\gamma$ is large enough and the ends of the strip need to be pulled to close up the bistrip. The observed buckling mode is similar to the ridge mode predicted by the linear stability analysis: the dihedral angle clearly varies along the central fold in part (c2) of the figure, and the centerline remains planar. The observed mode corresponds to an azimuthal wavevector $n=2$, as predicted by the theory.

Another buckling mode is observed in the experiments, which could not be anticipated based on the linear stability analysis. This mode, shown in the bottom row (a3-c3) in figure 8 is a non-planar pattern having a non-constant dihedral angle. A striking feature is that the deformation is localized at two opposite points, where the curvature is quite large. This mode is obtained for slightly larger values of $\gamma$ than the ridge mode, i.e. for an even larger overcurvature. This localized pattern is essentially non-linear, and will be explained later on in section 7 .

In figure 9, we show that it is possible to force the bistrip into a higher centerline mode, $n=3$. Starting from the natural buckling mode $n=2$, in figure 8 (c1), the higher mode can be obtained by 

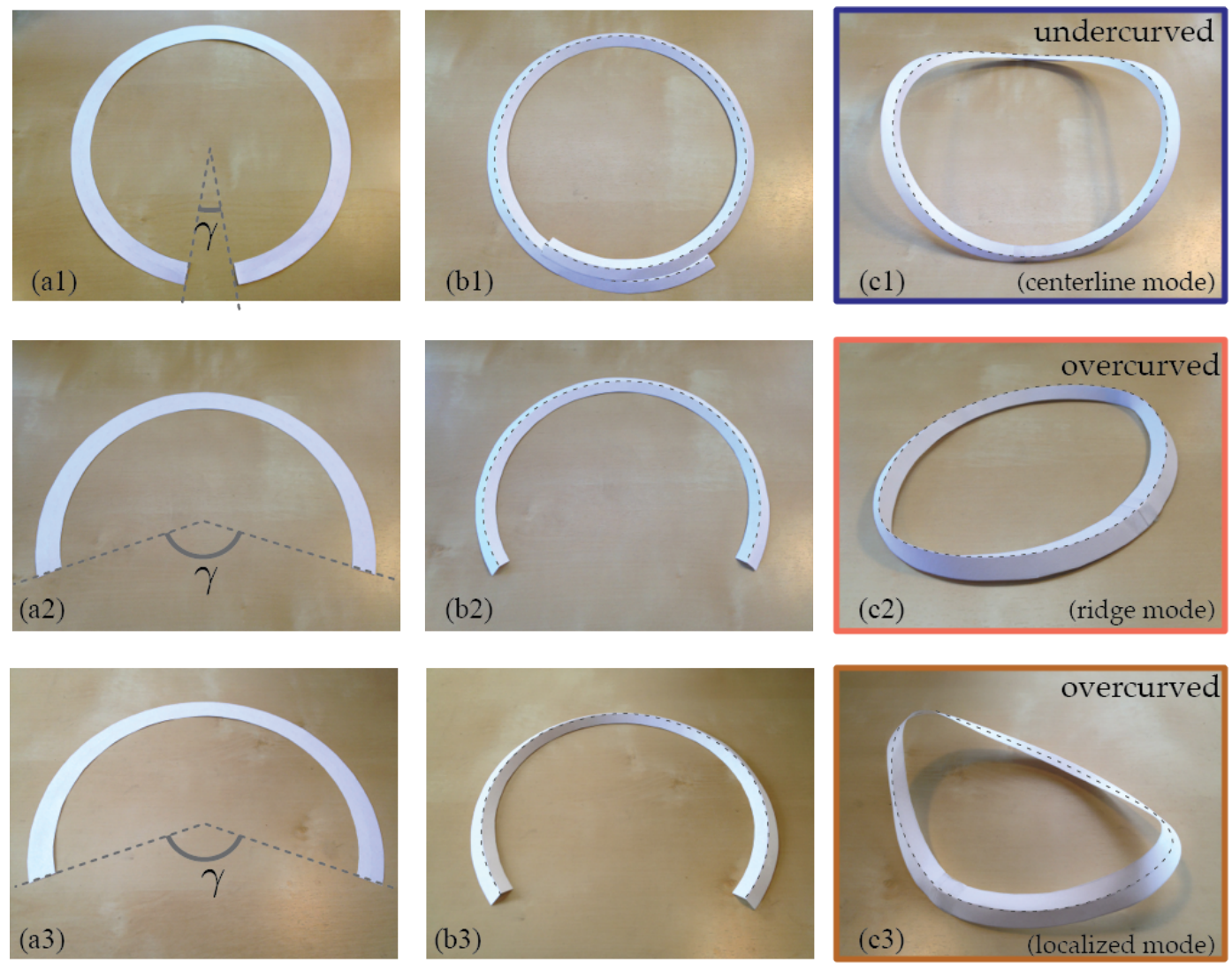

Figure 8: Observation of the centerline buckling mode (top row) and ridge buckling mode (bottom row) in a paper model. Both modes have $n=2$ bumps, as predicted by the linear stability analysis. The bistrip is prepared as explained in figure 5: (a) an annular region is cut out in a piece of paper and a sector of variable angular size $\gamma$ is removed from it; (b) the central ridge is pleated, leading to an increase in the curvature of the ridge, hence an overlap of the two free ends (undercurved case, b1) or a reduction of the gap between them (overcurved case, b2); (c) gluing the free ends together makes the bistrip buckle. In (b) and (c), the position of the ridge is highlighted by a dashed overlay. 


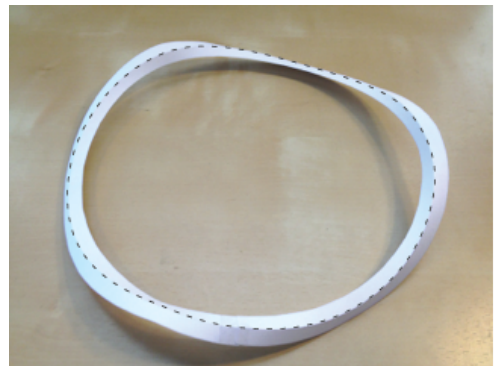

(a)

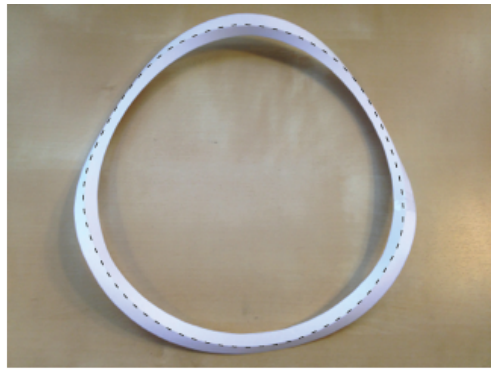

(b)

Figure 9: A higher-order centerline mode, with a wavenumber $n=3$, viewed from two angles. This mode is achieved by compressing the natural mode $n=2$ between two plates.
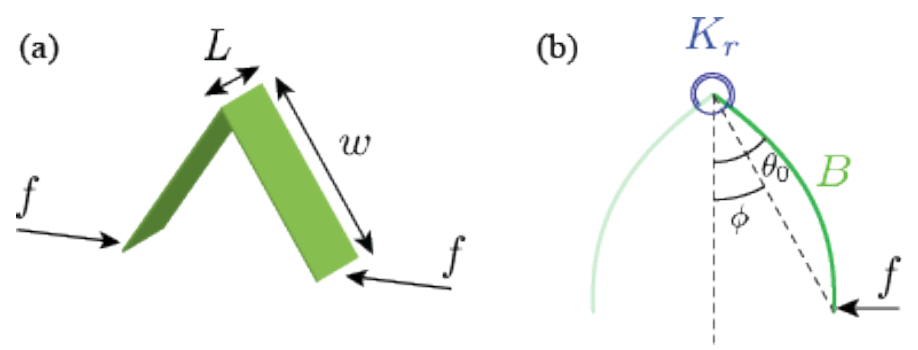

Figure 10: Sketch of the pinching experiment used to measure the dimensionless ridge stiffness $\bar{K}_{r}$.

squeezing the paper model between two parallel plates. When released, the shape with $n=3$ bumps appears to be stable: it is likely to be a local equilibrium configuration.

\subsection{Measuring the ridge stiffness}

Here we show how the dimensionless ridge stiffness $\bar{K}_{\mathrm{r}}$ can be measured experimentally. The value of $\bar{K}_{\mathrm{r}}$ is required to produce the post-buckled solution in the following section. The experimental set up is depicted in figure 10. We cut out a short segment of the bistrip, with axial length $L=1 \mathrm{~cm}$. The length $L$ and width $w=2 \mathrm{~cm}$ are comparable, and are much larger than the thickness $h \sim 0.2 \mathrm{~mm}$. As sketched in the figure, a pinching force $f$ is applied at the endpoints of the flaps. By measuring how much the flaps bend in response to this force, versus how much the dihedral changes, one can find out the value of $\bar{K}_{\mathrm{r}}$.

To do so, we measured experimentally the values of the dihedral angle $\theta_{0}=\frac{\pi}{2}-\beta$ and of the angle $\phi$ made by the two endpoints (see figure) for various values of the applied force. We simulated the problem of a 2D Elastica attached to an elastic hinge numerically. This problem depends only on the dimensionless stiffness $\hat{K}_{\mathrm{r}}=\frac{w^{2} K_{\mathrm{r}}}{B}$. We plotted several parametric curves $f \mapsto\left(\theta_{0}\left(\hat{K}_{\mathrm{r}}, f\right), \phi\left(\hat{K}_{\mathrm{r}}, f\right)\right)$ corresponding to different values of $\hat{K}_{\mathrm{r}}$. The experimental datapoints were found to be distributed along one of the simulation curves, and this allowed the parameter $\hat{K}_{\mathrm{r}}$ to be determined. This parameter was then converted into the original dimensionless stiffness $\bar{K}_{\mathrm{r}}$ defined in equation $(79)$ using the formula $\bar{K}_{\mathrm{r}}=\left(r_{0} / w\right)^{2} \hat{K}_{\mathrm{r}}$. For the bistrips paper models used in the present paper, this yields $\bar{K}_{\mathrm{r}}=155$.

\section{Post-buckled solutions}

In this section, we investigate the post-buckled configurations of a bistrip numerically, by solving the non-linear equations using a continuation method. The goal is to provide an example of application of the bistrip model of section 3 in a fully non-linear setting, to validate the assumptions and the predictions of the linear stability analysis of section 5 , and to investigate the nature of the bifurcations. We would also like to explain the localized pattern observed in the experiments, which the linear stability analysis 
could not reproduce.

The continuation method is implemented in two steps: the symbolic calculation language Mathematica [39] is used to transform the equations for the strip in a set of first-order differential equations, and export the right-hand sides as computer code in the $\mathrm{C}$ language; in a second step, this code is used by the continuation software AUTO-07p [40] to produce the branches of equilibrium.

The unknowns are collected into a state vector $\underline{\mathcal{X}}(s)$,

$$
\underline{\mathcal{X}}(s)=\left\{\underline{R}(s), \underline{D}_{\mathbb{I I}}(s), \underline{D}_{I I}(s), \underline{N}(s), \beta(s), \beta^{\prime}(s), \Omega_{\mathbb{I I}}(s), \Lambda_{+}(s), \Lambda_{-}(s)\right\},
$$

whose dimension is $N=17$. The numerical continuation method requires that we write the non-linear equations of equilibrium for the bistrip in the form of $N$ first-order ordinary differential equations,

$$
\underline{\mathcal{X}}^{\prime}(s)=\underline{\Phi}(\underline{\mathcal{X}}(s))
$$

together with $N$ boundary conditions,

$$
\underline{\Gamma}(\underline{\mathcal{X}}(0), \underline{\mathcal{X}}(L))=\underline{0} .
$$

Let us now explain how the equilibrium equations for the bistrip are cast in this form, starting with the differential equation 84a). In terms of $\mathcal{X}(s)$, the following quantities are first reconstructed: $\underline{D}_{I}=\underline{D}_{I I} \times \underline{D}_{\text {III }}, \Omega_{\mathbb{I I}}=\kappa_{\mathrm{g}} / \cos \beta, \underline{\Omega}=\sum_{\mu=I I}^{\text {III }} \Omega_{\mu} \underline{D}_{\mu}$. Then, the derivative of $\mathcal{X}(s)$ is calculated as follows: $\underline{R}^{\prime}=\underline{D}_{I I}, \underline{D}_{\text {III }}^{\prime}=\underline{\Omega} \times \underline{D}_{\mathbb{I I}}, \underline{D}_{I I}^{\prime}=\underline{\Omega} \times \underline{D}_{I I}, \underline{N}^{\prime}=\underline{0}$; the derivative of $\beta$ is directly equated to the following state variable $\beta^{\prime}$; by inserting the full constitutive law (39) into the global balance of moments $\left.42 \mathrm{~b}\right)$ and the equilibrium equation for the ridge (35), we obtain four scalar equations, which we solve symbolically for $\beta^{\prime \prime}, \Omega_{\mathbb{I I I}}^{\prime}, \Lambda_{+}^{\prime}$ and $\Lambda_{-}^{\prime}$. These expressions for $\left\{\underline{R}^{\prime}, \underline{D}_{\text {III }}^{\prime}, \cdots, \Lambda_{-}^{\prime}\right\}$ are collected into a vector $\underline{\Phi}(\underline{\mathcal{X}}(s))$ of length $N=17$, and the map $\underline{\Phi}$ is implemented numerically in the C language.

The vector of the boundary conditions $\underline{\Gamma}$ is constructed as follows. We note that the solution is defined up to a rigid-body motion, and remove this indeterminacy by the convention $\underline{R}(\underline{0})=\underline{0}, \underline{D}_{I I}(0)-\underline{e}_{x}=$ $\underline{0}, \underline{D}_{I I}(0)-\underline{e}_{y}=\underline{0}$. We also enforce the periodicity conditions $\underline{R}(L)-\underline{R}(0)=\underline{0}, \beta(L)-\beta(0)=0$, $\beta^{\prime}(L)-\beta^{\prime}(0)=0,\left(\underline{D}_{I I I}\right)_{y}(L)-\left(\underline{D}_{I I I}\right)_{y}(0)=0,\left(\underline{D}_{I I I}\right)_{z}(L)-\left(\underline{D}_{I I I}\right)_{z}(0)=0,\left(\underline{D}_{I I}\right)_{z}(L)-\left(\underline{D}_{I I}\right)_{z}(0)=0$. This yields a total of $N=17$ scalar boundary conditions, which are implemented as a map $\underline{\Gamma}(\underline{\mathcal{X}}(0), \underline{\mathcal{X}}(L))$ in the $\mathrm{C}$ language. It can be checked that these periodicity conditions are necessary and sufficient to warrant the periodicity of all the physical quantities of the strip, such as $\Omega_{\mu}, \underline{D}_{I I}, \Lambda_{ \pm}$, etc.

We work in a set of units such that $r_{0}=1$, i.e. the curvilinear length of the ridge is $L=2 \pi$, and the bending modulus of the flaps is $B=1$. The parameters of the simulation are the natural angle $\beta_{\mathrm{n}}$ of the ridge, the ridge stiffness $K_{\mathrm{r}}$ (which coincides with the rescaled one, $\bar{K}_{\mathrm{r}}$, in this set of units), and the ridge angle $\beta_{0}$ in the circular configuration. The geodesic curvature $\kappa_{\mathrm{g}}=\cos \beta_{0}$ is viewed as a dependent variable (see equation (52)). We only consider the fundamental buckling modes, $n=2$.

The boundary value problem in equations (84) is solved using AUTO-07p. A branch of solutions is produced by starting from the circular configuration, with a radius $r_{0}=1$ and a ridge angle $\beta_{0}$. The natural value of the ridge angle is initialized to $\beta_{\mathrm{n}}=\beta_{0}$, and then used as a continuation parameter: this mimics the act of creasing the central fold further $\left(\beta_{\mathrm{n}}>\beta_{0}\right)$, or flattening it $\left(\beta_{\mathrm{n}}<\beta_{0}\right)$. The equilibrium branches are followed as $\beta_{\mathrm{n}}$ is varied. Bifurcation diagrams obtained in this way are shown in figure 11 . The parameter $\beta_{0}$ and $\bar{K}_{\mathrm{r}}$ were set to the values corresponding to our experiments. In the diagram, we use the buckling indicators $I_{\mathrm{c}}$ and $I_{\mathrm{r}}$ for the centerline and for the ridge modes, respectively. They are defined by

$$
I_{\mathrm{c}}=\left\langle\Omega_{\mathrm{III}^{2}}\right\rangle^{1 / 2}, \quad I_{\mathrm{r}}=\left\langle{\beta^{\prime}}^{2}\right\rangle^{1 / 2},
$$

where $\langle f\rangle=\frac{1}{L} \int_{0}^{L} f(s) \mathrm{d} s$ denotes the average of a function $f$. These definitions are motivated by our stability analysis: the centerline mode is a pure twist mode $\left(I_{\mathrm{c}} \neq 0\right.$ and $\left.I_{\mathrm{r}}=0\right)$, while the ridge mode is a pure bending mode $\left(I_{\mathrm{c}}=0\right.$ and $\left.I_{\mathrm{r}} \neq 0\right)$. 


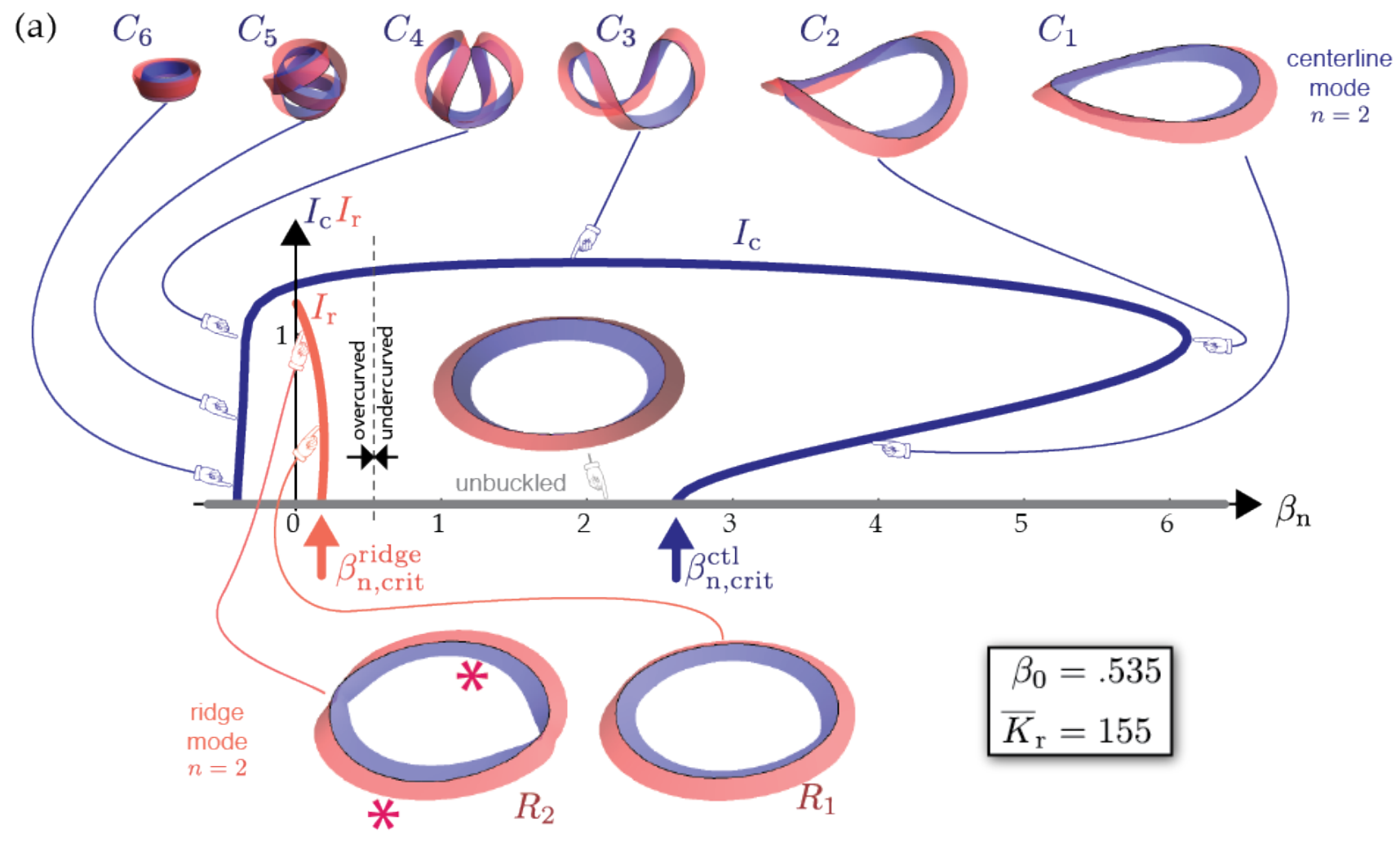

(b)

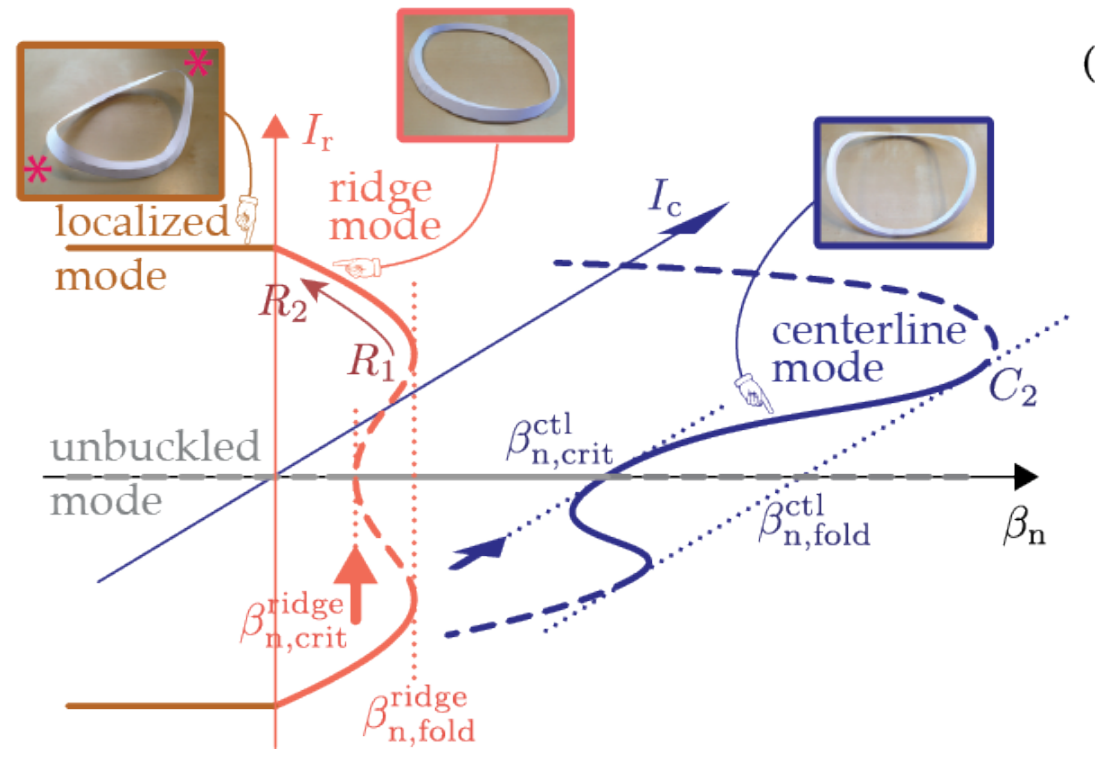

(c)

Figure 11: Non-linear branches of equilibrium obtained by solving the fully non-linear equations of section 3.7. (a) Results of the numerical continuation using AUTO for $\bar{K}_{\mathrm{r}}=155$ and $\beta_{0}=.535$ (typical values of our experiments). On the unbuckled branch (grey), both $I_{\mathrm{r}}$ and $I_{\mathrm{c}}$ cancel; on the ridge branch (orange), only $I_{\mathrm{r}}$ is non-zero; on the centerline branch (dark blue), only $I_{\mathrm{c}}$ is non-zero. Only the buckled branches with azimuthal wavenumber $(n=2)$ are shown. The thick arrows below the $\beta_{\mathrm{n}}$ axis show the critical loads predicted by the linear stability analysis of section 5. (b) Stylized view of the same diagram representing the ridge and centerline modes in perpendicular directions, extending the branches to $I_{\mathrm{r}}<0$ and $I_{\mathrm{c}}<0$ by symmetry, stretching the ridge branch horizontally for better legibility, and showing the stable (solid curves) and unstable (dashed curves) portions of the branches. (c) Plot of the dihedral angle $\beta$ as a function of the arclength $s$ for different solutions along the ridge branch: the dihedral angle progressively localizes as one moves towards the endpoint $R_{2}$ of the branch. There, the bistrip flattens $(\beta=0)$ at two opposite points (red asterisks). Beyond this point $R_{2}$, for negative values of $\beta_{\mathrm{n}}$, the ridge branch connects to localized modes (brown curve in part b of the figure). 
A stylized version of the numerical diagram obtained in figure 11(a) is shown in figure 11(b): in the latter, the ridge branch (orange curve) has been stretched for better legibility. First, we note that the buckling thresholds predicted by the linear stability analysis of section 5 are correct: they are shown by the thick arrows below the $\beta_{\mathrm{n}}$ axis in part (a) of the figure, and correspond exactly to the values of $\beta_{\mathrm{n}}$ where the centerline or ridge branch meet the unbuckled branch. These buckling thresholds read, from equations $\sqrt{78}$ and $82, \beta_{\mathrm{n}, \text { crit }}^{\text {ridge }}\left(\bar{K}_{\mathrm{r}}, \beta_{0}, n\right)=0.177$ and $\beta_{\mathrm{n}, \mathrm{crit}}^{\text {ridge }}\left(\bar{K}_{\mathrm{c}}, \beta_{0}, n\right)=2.612$, with $\bar{K}_{\mathrm{r}}=155, \beta_{0}=.535$ and $n=2$. For reference, the transition from the overcurved to the undercurved case, which can be found by solving $M_{I I}^{0}=0$ for $Q_{0}$ in equation (55) and then for $\beta_{\mathrm{n}}$ in the ridge's constitutive law, occurs at the intermediate value $\beta_{\mathrm{n}}=0.537$, as indicated by the dashed vertical line in the figure (we noted earlier that the centerline mode occurs in the undercurved case and the ridge mode in the overcurved case). The agreement of the non-linear post-buckling and linear stability analyses on the initial thresholds confirms the relevance of the simplified constitutive law (\$3.8) to the linear stability analysis.

Near $\beta_{\mathrm{n}, \text { crit }}^{\text {ridge }}$, the centerline branch emerges through a continuous pitchfork bifurcation. The part of the branch that extends between this initial bifurcation and the limit point $C_{2}$ is stable, see part (b) of the figure. This branch spans the interval $\beta_{\mathrm{n} \text {,crit }}^{\text {ridge }} \leq \beta_{\mathrm{n}} \leq \beta_{\mathrm{n} \text {,fold }}^{\text {ridge }}$, where the value of $\beta_{\mathrm{n}}$ corresponding to the limit point $C_{2}$ is $\beta_{\mathrm{n} \text {,fold }}^{\text {ridge }}=6.120$. The presence of this stable branch is consistent with the experimental observation of saddle-like shapes for large enough undercurvature, as in the experimental snapshot framed in dark blue in part (a) of the figure. Note that the stability of the equilibria has been inferred by comparing the elastic energy of the various types of solutions for a given value of $\beta_{\mathrm{n}}$; a detailed analysis of stability would be needed to confirm this. Past the limit point $C_{2}$, the centerline branch is unstable. This unstable branch ultimately connects with 3 -fold circular solutions, see snapshot $C_{6}$ in the figure. We ignore the self-contact that starts to take place beyond configuration $C_{4}$.

Note that for the particular values $\bar{K}_{\mathrm{r}}=155$ and $\beta_{0}=.535$ used to generate figure 11 , the stable centerline branch lies above $\beta_{\mathrm{n}, \text { crit }}^{\text {rige }}=2.612$. This value is beyond the maximum value $\beta_{\mathrm{n}}=\pi / 2$ allowed by the non-penetration condition of the flaps. We conclude that for these specific values of the parameters, the centerline buckling mode cannot be observed. The experimental snapshots of centerline modes shown in figure 11(b) and 8(c1) were indeed obtained for a much lower value of the angle $\beta_{0}$, and the corresponding buckling thresholds $\beta_{\mathrm{n}, \text { crit }}^{\text {ctl }}$ predicted by equation 78 are below $\pi / 2$.

We now examine the ridge branch. As emphasized in part (b) of the figure, this branch is produced by a discontinuous pitchofork bifurcation: the weakly post-buckled ridge solutions exist for values of $\beta_{\mathrm{n}}$ lying on the same side of the initial threshold, $\beta_{\mathrm{n}}>\beta_{\mathrm{n} \text {,crit }}^{\text {ridge }}$, as the stable unbuckled configuration. This implies that the portion of the ridge branch between the initial bifurcation threshold $\beta_{\mathrm{n}, \mathrm{rit}}^{\text {rit }}$ and the fold point corresponding to the configuration $R_{1}$, which occurs for $\beta_{\mathrm{n}}=\beta_{\mathrm{n} \text {,fold }}^{\text {ridge }}=0.196$, is unstable: see dashed curve in part (b) of the figure. Further along the ridge branch, beyond the fold point $R_{1}$, it becomes stable. In the interval $\beta_{\mathrm{n}, \text { crit }}^{\text {ridge }}=0.177<\beta_{\mathrm{n}}<\beta_{\mathrm{n} \text {,fold }}^{\text {ridge }}=0.196$, both the ridge mode and the unbuckled solution are stable; this interval has been stretched in figure 11(b) to improve legibility, but it is actually quite small. The ridge mode ceases to exist when $\beta_{\mathrm{n}}$ reaches a numerical value equal to zero within numerical accuracy, which happens slightly beyond the configuration labelled $R_{2}$ in the figure. The plot of the ridge angle $\beta(s)$ in figure 11(c) shows that the branch ends when the ridge angle, which progressively concentrates into two narrow peaks, reaches the value $\beta=0$ at two opposite points (asterisks). The solutions that exist past this point cannot be described with our equations, as we explicitly assumed $\beta \neq 0$ to derive equation (31). They can nevertheless be discussed as follows. When the ridge becomes flat, $\beta=0$, the bistrip suddenly acquires another degree of freedom, which involves bending both flaps into a cylindrical shape, with generatrices locally perpendicular to the fold line. This is exactly what happens in the localized mode observed in the experiments, see the asterisks in the experimental picture framed in brown in figure 11(b). Therefore, we infer that the ridge branch connects to a branch made of localized solutions in the region $\beta_{\mathrm{n}}<0$, sketched by the brown line in part (b) of the figure. This is consistent with the experimental fact that planar ridge solutions, which are rarely observed as they exist in a narrow interval of $\beta_{\mathrm{n}}$, evolve into non-planar localized solutions when the amount of overcurvature 
is increased.

Overall, the post-buckling diagram explains the three types of patterns observed in the experiments. The localized pattern could not be anticipated by the linear stability analysis as it is produced by a secondary bifurcation: along the ridge branch the dihedral angle $\beta$ progressively concentrates until it reaches zero at two opposite points, allowing the bistrip to become suddenly non-planar.

\section{Conclusion}

We have considered the large deformations of thin elastic strips, whose width $w$ is much smaller than its length $L$ but much larger than its thickness $h: h \ll w \ll L$. For thin beams having a slender crosssection, $h \ll w$, the classical rod theory of Kirchhoff is known to be inapplicable. Such beams are usually modeled using Vlasov's theory for thin-walled beams. Vlasov's models can be justified from 3D elasticity but only in the case of moderate deformations, when the cross-sections bend by a small amount. In the present work, however, we have considered large deformations of thin strips. The strip has been modeled as an inextensible plate, and the geometric constraint of inextensibility has been treated exactly: the cross-sections are allowed to bend by a significant amount. Our model extends the classical strip model of Sadowsky, and reformulate it in a way that fits into the classical theory of rods.

To do this, we have identified the applicable geometrical constraints and constitutive law. The latter is non-linear because of underlying constraint of developability. The other classical equations for thin rods are applicable (inextensibility and unshearability constraints, geometric definition of the twist-curvature strain, equations of equilibrium). Unifying the description of of elastic strips and rods allows the large body of numerical and analytical methods developed for rods, to be ported to strips: our stability analysis of the bistrip was adapted from the classical stability analyses of elastic rings.

For the purpose of illustration, our model has been applied to a specific geometry: the equilibria of a closed bistrip have been analyzed. Bifurcated solutions reported in prior work have been interpreted based on a instability affecting the circular solutions. Two other, novel types of patterns have been demonstrated in experiments. We have identified the residual bending moment in the circular configuration as the stress driving these instabilities. The sign of this residual stress has been shown to determine which buckling mode occurs. A symmetry argument has been invoked to explain the main features of these buckling modes. The selection of the wavenumber of the modes has been accounted for. The non-linear features of the instability have been explored numerically using a continuation method. In particular, we have identified a localized mode, that can only be interpreted based on the post-buckling analysis.

In future work, it would be interesting to extend the present approach to corrugated shells. Such shells are obtained by folding an elastic plate along a family of folds that are locally parallel to each other. The presence of the folds has a dramatic influence on the mechanical behavior of the structure: for instance, they can make it behave like a hyperbolic shell, and can couple to two modes of bending [41. These interesting behaviors have awaken a marked interest recently. So far, the analysis of corrugated shells has been mainly carried out at the geometric level [42, 43] or for specific fold geometries [44, 45]. Generalizing the approach followed in our paper, it should be possible to account for the presence of the folds through an effective (homogenized) constitutive law. This would make it possible to bridge the gap between the literature on the mechanics of elastic shells, and the young field of corrugated shells.

\section{A Constitutive law for an elastic rod with constraints}

In this appendix, we derive the general expression of the constitutive law for an elastic rod subjected to kinematical constraint, and possessing an internal degree of freedom. It is considered inextensible and the arclength is denoted by $s$. A configuration of the rod is parameterized by its centerline $\underline{r}(s)$, by an orthonormal material frame $\underline{d}_{i}(s)$ with $i=1,2,3$, and by an internal variable $k(s)$ (in the elastic strip model, this internal variable is the transverse curvature $k_{11}$ ). These functions have to satisfy the 
Euler-Bernoulli and inextensibility constraints in equation (1, 2). The Darboux vector $\underline{\omega}(s)$ is defined by equation (3), and we denote by $\omega_{i}(s)=\underline{\omega}(s) \cdot \underline{d}_{i}(s)$ its components in the material frame. We assume that the elastic energy $E_{\text {el }}$ of the rod is the integral of a density of elastic energy, which is itself a function of the local twist and curvature strains $\left(\omega_{1}, \omega_{2}, \omega_{3}\right)$ and of the internal parameter $k$. In addition to the inextensibility and Euler-Bernoulli constraints, the rod is subjected to a kinematical constraint which writes $\mathcal{C}\left(\omega_{i}(s), k(s)\right)=0$.

The rod is subjected to a density of external force $\underline{p}(s)$ and a density of external moment $\underline{q}(s)$ per arc-length $\mathrm{d} s$. Its equilibrium is governed by the principle of virtual work. The latter states that, for any virtual motion (see for instance [46, 22]),

$$
\begin{aligned}
-\int\left(\sum_{i=1}^{3} \frac{\delta E_{\mathrm{el}}}{\delta \omega_{i}} \delta \omega_{i}+\frac{\delta E_{\mathrm{el}}}{\delta k} \delta k\right) \mathrm{d} s+\int \lambda\left(\sum_{i=1}^{3} \frac{\partial \mathcal{C}}{\partial \omega_{i}} \delta \omega_{i}+\frac{\partial \mathcal{C}}{\partial k} \delta k\right) \mathrm{d} s \cdots \\
+\int \underline{n} \cdot\left(\delta \underline{r}^{\prime}-\delta \underline{d}_{3}\right) \mathrm{d} s+\int(\underline{p} \cdot \delta \underline{r}+\underline{q} \cdot \underline{\delta \theta}) \mathrm{d} s=0
\end{aligned}
$$

where $\delta E_{\mathrm{el}} / \delta \omega_{i}$ and $\delta E_{\mathrm{el}} / \delta k$ denote the functional derivative of the elastic energy $E_{\mathrm{el}}$ with respect to $\omega_{i}(s)$ and $k(s)$, respectively. In this equation, virtual (infinitesimal) quantities are prefixed with the letter $\delta: \delta \underline{r}, \delta \underline{d}_{3}, \delta \underline{\theta}, \delta \omega_{i}, \delta k$ are the virtual change of centerline, of tangent, the virtual infinitesimal rotation, the virtual increment of twist and curvature, and of internal parameter, respectively. The first term in equation 86 is the virtual internal work which represents the elastic stress in the rod: as usual in the elastic case, it is the opposite of the first variation of the elastic energy, $-\delta E_{\mathrm{el}}$. The second term takes into account geometric constraint, $\mathcal{C}$ and $\lambda(s)$ is the associated Lagrange multiplier. The third term is associated with kinematic constraint in equation (1); the corresponding Lagrange multiplier $\underline{n}(s)$ can be interpreted as the internal force. The last term is the virtual external work. Note that in the particular case where the external load is conservative, the principle of virtual work (86) expresses the condition of stationarity of the total energy subjected to the three kinematic constraints listed above, as obtained by Lagrange's method of constrained variations.

Cancelling the term proportional to $\delta k$ in equation (86), we obtain the condition of equilibrium with respect to the internal variable as

$$
-\frac{\delta E_{\mathrm{el}}}{\delta k}+\lambda \frac{\partial \mathcal{C}}{\partial k}=0
$$

Next, the strain increments are combined into a single vector defined by $\delta \underline{\omega}=\sum_{i=1}^{3} \delta \omega_{i} \underline{d}_{i}$, which can be interpreted as the gradient of virtual rotation, $\delta \underline{\omega}=\underline{\delta \theta^{\prime}}$, see [22]. We also define

$$
\underline{m}=\sum_{i=1}^{3}\left(\frac{\delta E_{\mathrm{el}}}{\delta \omega_{i}}-\lambda \frac{\partial \mathcal{C}}{\partial \omega_{i}}\right) \underline{d}_{i} .
$$

This allows us to rewrite the principle of virtual work in equation $(86)$ as

$$
-\int \underline{m} \cdot \underline{\delta \theta^{\prime}} \mathrm{d} s+\int \underline{n} \cdot\left(\delta \underline{r}^{\prime}-\underline{\delta \theta} \times \underline{r}^{\prime}\right) \mathrm{d} s+\int(\underline{p} \cdot \delta \underline{r}+\underline{q} \cdot \underline{\delta \theta}) \mathrm{d} s=0 .
$$

This is the classical expression of the principle of virtual work for inextensible Euler-Bernoulli rods without additional constraints. By using geometrical identities and by integrating by parts, one can show [36, 46, 22 that the corresponding equations of equilibrium in strong form are the Kirchhoff equations (13).

We conclude than the equilibrium of a constrained elastic rod having an internal degree of freedom $k(s)$ is governed by the equilibrium of the internal degree of freedom in equation (87a) and by the classical Kirchhoff equation for the equilibrium of rods. In the latter, one must use the expression of the internal moment $\underline{m}(s)$ given by the constitutive law $87 \mathrm{~b})$. Equations $87 \mathrm{a}$ and $87 \mathrm{~b}$ are the main results of this appendix. 


\section{B Symmetry relevant to the planar, circular state}

We identify a symmetry of the equations of equilibrium for a bistrip. By this symmetry, the centerline gets reflected through to a plane. The ridge does not get flipped, however: this symmetry is not merely a pointwise reflection of the entire bistrip. This symmetry accounts for the two family of buckling modes from the circular configuration (the centerline and ridge modes): this modes are the eigenvectors of the symmetry operator.

\section{B.1 Definition of the symmetry}

Let us denote $\underline{G}$ the reflection through the $(x y)$ plane in the Euclidean space, $\underline{G} \cdot(x, y, z)=(x, y,-z)$. We consider a solution $\mathcal{S}$ of the equilibrium problem for the bistrip, as summarized in section 3.7 . This solution is specified by the functions

$$
\mathcal{S}=\left(\underline{R}, \underline{D}_{I}, \underline{D}_{I I}, \underline{D}_{I I}, \beta, \underline{\Omega}, \Lambda_{+}, \Lambda_{-}, \underline{N}, \underline{M}, \underline{\Delta}\right) .
$$

The symmetry is defined by its action onto the space of configurations: it maps $\mathcal{S}$ onto another configuration $\tilde{\mathcal{S}}=\left(\underline{\tilde{R}}, \underline{\tilde{D}}_{I}, \cdots\right)$ defined by

$$
\begin{aligned}
& \underline{\tilde{R}}(s)=\underline{G} \cdot \underline{R}(s) \\
& \underline{\tilde{D}}_{\mu}(s)=-\eta_{\mu} \underline{\underline{G}} \cdot \underline{D}_{\mu}(s) \\
& \tilde{\beta}(s)=\beta(s) \\
& \underline{\tilde{\Omega}}(s)=-\underline{\underline{G}} \cdot \underline{\Omega}(s) \\
& \tilde{\Lambda}_{ \pm}(s)= \pm \Lambda_{ \pm}(s) \\
& \underline{\tilde{N}}(s)=\underline{G} \cdot \underline{N}(s) \\
& \underline{\tilde{M}}(s)=-\underline{\underline{G}} \cdot \underline{M}(s) \\
& \tilde{\Delta}(s)=\underline{\underline{G}} \cdot \underline{\Delta}(s),
\end{aligned}
$$

where $\mu \in\{I, I I, I I\}$, and $\eta_{\mu}$ is the sign defined by

$$
\eta_{\mu}=(-1)^{\mu}= \begin{cases}-1 & \text { for } \mu=I, \text { III } \\ +1 & \text { for } \mu=I I .\end{cases}
$$

By equation (90b), the directors basis is mapped to $\underline{\tilde{D}}_{I}=+\underline{\underline{G}} \cdot \underline{D}_{I}, \underline{\tilde{D}}_{\mathbb{I}}=-\underline{\underline{G}} \cdot \underline{D}_{I I}$ and $\underline{\tilde{D}}_{\mathbb{I I}}=+\underline{\underline{G}} \cdot \underline{D}_{\mathbb{I I}} \cdot$ The minus sign in the definition of $\underline{\tilde{D}}_{I I}$ preserves the right-handedness of the frame.

The components of the Darboux vector in the local frame are transformed according to $\tilde{\Omega}_{\mu}=\underline{\tilde{\Omega}} \cdot \underline{\tilde{D}}_{\mu}=$ $(-\underline{\underline{G}} \cdot \underline{\Omega}) \cdot\left(-\eta_{\mu} \underline{\underline{G}} \cdot \underline{D}_{\mu}\right)=\eta_{\mu} \underline{\Omega} \cdot \underline{D}_{\mu}=+\eta_{\mu} \Omega_{\mu}$. This implies $(o) \tilde{\Omega}_{I}=\Omega_{I}=0$ : the symmetric configuration satisfies the constraint (41c), and (ii) the unconstrained curvatures transform according to

$$
\begin{aligned}
& \tilde{\Omega}_{\text {II }}=+\Omega_{\text {II }} \\
& \tilde{\Omega}_{\text {III }}=-\Omega_{\text {III }} .
\end{aligned}
$$

By a similar argument, the internal moments $\underline{M}$ and $\underline{\Delta}$ are transformed according to $\tilde{M}_{j}=+\eta_{j} M_{j}$ and $\tilde{\Delta}_{j}=-\eta_{j} \Delta_{j}$. Using these transformation rules, it can be checked that the new state $\tilde{\mathcal{S}}$ satisfies the equilibrium equations for a bistrip summarized in section 3.7, when $\mathcal{S}$ is itself an equilibrium solution. Note that an external loading may break this symmetry, unless it is itself symmetric — in this paper, we ignore the external loading. 


\section{Acknowledgements}

MAD would like to thank financial support from NSF DMR 0846582 and NSF-supported MRSEC on Polymers at UMass (DMR-0820506) during the initial stages of this project.

\section{References}

[1] H. M. Wingler, Bauhaus: Weimar, Dessau, Berlin, Chicago, The MIT Press, 1969.

[2] H. Engel, Structure systems, Westport, CT: Praeger, 1968.

[3] P. Jackson, Folding Techniques for Designers: From Sheet to Form, Laurence King Publishers, 2011.

[4] M. Schenk, S. D. Guest, Origami folding: A structural engineering approach, in: P. Wang-Iverson, R. J. Lang, M. Yim (Eds.), Origami ${ }^{5}$, Fifth International Meeting of Origami Science, Mathematics, and Education, A K Peters/CRC Press, 2011, pp. 291-303.

[5] K. Miura, Method of packing and deployment of large membranes in space, The Institute of Space and Astronautical Science.

[6] K. Kuribayashi, K. Tsuchiya, Z. You, D. Tomus, M. Umemoto, T. Ito, M. Sasaki, Self-deployable origami stent grafts as a biomedical application of Ni-rich TiNi shape memory alloy foil, Materials Science and Engineering: A 419 (1-2) (2006) 131-137.

[7] D. A. Huffman, Curvature and Creases: A Primer on Paper, IEEE Transactions on Computers C-25 (10) (1976) 1010-1019.

[8] J. P. Duncan, J. L. Duncan, Folded Developables, Proceedings of the Royal Society of London. Series A 383 (1784) (1982) 191-205.

[9] D. Fuchs, S. Tabachnikov, More on Paperfolding, The American Mathematical Monthly 106 (1) (1999) 27-35.

[10] H. Pottmann, J. Wallner, Computational Line Geometry, Springer-Verlag, Berlin, Heidelberg, 2001.

[11] M. Kilian, S. Flöry, Z. Chen, N. J. Mitra, A. Sheffer, H. Pottmann, Curved folding, SIGGRAPH Computer Graphics 27 (3).

[12] M. A. Dias, L. H. Dudte, L. Mahadevan, C. D. Santangelo, Geometric Mechanics of Curved Crease Origami, Phys. Rev. Lett. 109 (11) (2012) 114301.

[13] M. Spivak, A comprehensive introduction to differential geometry, 2nd Edition, Vol. 3, Publish or perish, Inc., 1979.

[14] M. P. do Carmo, Differential Geometry of Curves and Surfaces, Prentice-Hall, Englewood Cliffs, 1976.

[15] E. H. Dill, Kirchhoff's theory of rods, Archive for history of exact sciences 44 (1992) 1-23.

[16] A. E. H. Love, A Treatise on the Mathematical Theory of Elasticity, Dover Publications, USA, 1944.

[17] S. S. Antman, Nonlinear Problems of Elasticity, Springer, 1995.

[18] C. W. Wolgemuth, R. E. Goldstein, T. R. Powers, Dynamic supercoiling bifurcations of growing elastic filaments, Physica D: Nonlinear Phenomena 190 (3-4) (2004) $266-289$. 
[19] D. Moulton, T. Lessinnes, A. Goriely, Morphoelastic rods. Part I: A single growing elastic rod, Journal of the Mechanics and Physics of Solids 61 (2) (2013) 398-427.

[20] Y. Shi, J. E. Hearst, The kirchhoff elastic rod, the nonlinear schrödinger equation, and dna supercoiling, The Journal of Chemical Physics 101 (6) (1994) 5186-5200.

[21] T. R. Powers, Dynamics of filaments and membranes in a viscous fluid, Reviews of Modern Physics 82 (2) (2010) 1607-1631.

[22] B. Audoly, Y. Pomeau, Elasticity and geometry: from hair curls to the nonlinear response of shells, Oxford University Press, 2010.

[23] R. E. Goldstein, P. B. Warren, R. C. Ball, Shape of a ponytail and the statistical physics of hair fiber bundles, Physical Review Letters 108 (2012) 078101.

[24] A. Hamdouni, O. Millet, An asymptotic non-linear model for thin-walled rods with strongly curved open cross-section, International Journal of Non-Linear Mechanics 41 (3) (2006) 396-416.

[25] A. Hamdouni, O. Millet, An asymptotic linear thin-walled rod model coupling twist and bending, International Applied Mechanics 46 (9) (2011) 1072-1092.

[26] E. H. Mansfield, Large-deflexion torsion and flexure of initially curved strips, Proceedings of the Royal Society of London. A. Mathematical and Physical Sciences 334 (1598) (1973) 279-298.

[27] K. A. Seffen, Z. You, S. Pellegrino, Folding and deployment of curved tape springs, International Journal of Mechanical Sciences 42 (2000) 2055-2073.

[28] F. Guinot, S. Bourgeois, B. Cochelin, L. Blanchard, A planar rod model with flexible thin-walled cross-sections. application to the folding of tape springs, International Journal of Solids and Structures 49 (2012) 73-86.

[29] L. Giomi, L. Mahadevan, Multi-stability of free spontaneously curved anisotropic strips, Proceedings of the Royal Society A: Mathematical, Physical and Engineering Science 468 (2138) (2012) 511-530.

[30] M. Sadowsky, Ein elementarer Beweis für die Existenz eines abwickelbares Möbiusschen Bandes und Zurückfürung des geometrischen Problems auf ein Variationsproblem, Sitzungsber. Preuss. Akad. Wiss. 22 (1930) 412-415.

[31] W. Wunderlich, über ein abwickelbares möbiusband, Monatshefte für Mathematik 66 (3) (1962) 276-289.

[32] E. L. Starostin, G. H. M. van der Heijden, Tension-Induced multistability in inextensible helical ribbons, Phys. Rev. Lett. 101 (2008) 084301.

[33] E. L. Starostin, G. H. M. van der Heijden, The shape of a Möbius strip, Nature Materials 6 (11) (2007) 563-567.

[34] C. Lanczos, The Variational Principles of Mechanics, University of Toronto Press, 1970.

[35] P.-O. Mouthuy, M. Coulombier, T. Pardoen, J.-P. Raskin, A. M. Jonas, Overcurvature describes the buckling and folding of rings from curved origami to foldable tents, Nature Communications 3 (2012) 1290.

[36] N. Chouaïeb, Kirchhoff's problem of helical solutions of uniform rods and stability properties, Ph.D. thesis, École polytechnique fédérale de Lausanne, Lausanne, Switzerland (2003).

[37] E. E. Zajac, Stability of two planar loop Elasticas, Journal of Applied Mechanics 29 (1962) 136-142. 
[38] A. Goriely, Twisted elastic rings and the rediscoveries of Michell's instability, Journal of Elasticity 84 (2006) 281-299.

[39] Wolfram Research, Inc., Mathematica edition: Version 9.0, champaign, IL (USA) (2012).

[40] E. J. Doedel, A. R. Champneys, T. F. Fairgrieve, Y. A. Kuznetsov, B. Sandstede, X. J. Wang, AUTO-07p: continuation and bifurcation software for ordinary differential equations, See http://indy.cs.concordia.ca/auto/ (2007).

[41] A. D. Norman, K. A. Seffen, S. D. Guest, Morphing of curved corrugated shells, International Journal of Solids and Structures 46 (7-8) (2009) 1624-1633.

[42] E. D. Demaine, M. L. Demaine, V. Hart, G. N. Price, T. Tachi, (non)existence of pleated folds: how paper folds between creases, Graphs and Combinatorics 27 (3) (2011) 377-397.

[43] K. A. Seffen, Compliant shell mechanisms, Philosophical transactions. Series A, Mathematical, physical, and engineering sciences 370 (1965) (2012) 2010-26.

[44] M. A. Dias, C. D. Santangelo, The shape and mechanics of curved-fold origami structures, Europhysics Letters 100 (5) (2012) 54005.

[45] Z. Wei, Z. Guo, L. Dudte, H. Liang, L. Mahadevan, Geometric Mechanics of Periodic Pleated Origami, arXiv1211.6396 (2012) 1-28.

[46] D. J. Steigmann, M. G. Faulkner, Variational theory for spatial rods, Journal of Elasticity 33 (1) (1993) 1-26. 